\title{
Room-temperature aqueous Suzuki-Miyaura cross-coupling reactions catalyzed via recyclable palladium@halloysite nanocomposite
}

\author{
Jumanah Hamdi, Alexis A. Blanco, Brooke Diehl, John B. Wiley and Mark L. Trudell* \\ Department of Chemistry and Advanced Materials Research Institute, University of New Orleans, New Orleans, \\ Louisiana 70148
}

\section{Supporting Information}

\begin{tabular}{|c|c|}
\hline & page \\
\hline Experimental Procedures & S2-S4 \\
\hline TEM Images of Pd@Hal nanocomposite materials from Table 1. & S5-S14 \\
\hline TEM Images for Recycling Study & S15-S16 \\
\hline $\begin{array}{l}\text { Characterization of cross-coupling products (6) } \\
\text { (mp and }{ }^{1} \mathrm{H} \text { NMR) }\end{array}$ & S17-S30 \\
\hline References for characterization data & S30 \\
\hline
\end{tabular}


Materials and Methods. All reactions were carried out in oven-dried glassware under a $\mathrm{N}_{2}$ atmosphere unless indicated otherwise. Halloysite clay was purchased from Sigma Aldrich. All other chemicals were purchased from Alfa Aesar, Sigma Aldrich and VWR. Halloysite was purchased from Sigma Aldrich. All chemicals were used as received without further purification. ${ }^{1} \mathrm{H}$ and ${ }^{13} \mathrm{C}$ NMR spectra were recorded at r.t. in DMSO- $d_{6}$ on a Bruker $400 \mathrm{MHz}$ instrument operating at a frequency of $300 \mathrm{MHz}$ for ${ }^{1} \mathrm{H}$ NMR and $75 \mathrm{MHz}$ for ${ }^{13} \mathrm{C} \mathrm{NMR}$. ${ }^{1} \mathrm{H}$ chemical shifts were referenced to the DMSO solvent signal $(2.50 \mathrm{ppm}) .{ }^{13} \mathrm{C}$ chemical shifts were referenced to the DMSO solvent signal (39.51 ppm). Pd $\mathrm{d}_{\mathrm{NP}}$ size was determined from TEM images that were obtained on a JEOL 2010 equipped with an EDAX genesis energy dispersive spectroscopy (EDS) system, operated at an accelerating voltage of $200 \mathrm{kV}$ and an emission current of $109 \mu \mathrm{A}$. ICPAES analysis of the Pd@Hal was performed by Galbraith Laboratories, Inc., Knoxville, TN.

\section{Synthesis of 4\% Pd@Hal.}

A solution of $\mathrm{Pd}(\mathrm{OAc})_{2}(60 \mathrm{mg}, 0.26 \mathrm{mmol})$ in deionized water $(10 \mathrm{~mL})$ was prepared in a $50 \mathrm{~mL}$ Erlenmeyer flask. A solution of L-sodium ascorbate $(1400 \mathrm{mg}, 7.0 \mathrm{mmol})$ in deionized water (15 $\mathrm{mL}$ ) was added to the palladium acetate solution followed by addition of a solution of trisodium citrate $(570 \mathrm{mg}, 2.7 \mathrm{mmol})$ in deionized water $(10 \mathrm{~mL})$. The combined solution was allowed to stir for $15 \mathrm{~min}$ at room temperature. During the first 10 minutes, the initial light orange color of the palladium acetate solution mixture turned black, indicating the formation of Pd nanoparticles. After $15 \mathrm{~min}$, no more color changes were observed, and the solution was left to rest at room temperature, open to air for $80 \mathrm{~min}$. Halloysite $(600 \mathrm{mg}, 2.0 \mathrm{mmol}$ ) was added to the $\mathrm{Pd}$ nanoparticle solution and the colloid mixture was stirred for $15 \mathrm{~min}$. The mixture was then allowed to rest at room temperature for $10 \mathrm{~min}$. The mixture was centrifuged $(6000 \mathrm{rpm})$ and the liquid was decanted away from the solid residue. The residue was washed with deionized water $(3 \times 15 \mathrm{~mL})$ and isopropyl alcohol $(3 \times 15 \mathrm{~mL})$. The resultant powder was dried at room temperature for $24 \mathrm{~h}$ in a desiccator $\left(\mathrm{CaSO}_{4}\right)$ to afford 4\% Pd@Hal as a gray powder (620 mg).

\section{Synthesis of 25\% Pd@Hal.}

A solution of $\mathrm{Pd}(\mathrm{OAc})_{2}(140 \mathrm{mg}, 0.60 \mathrm{mmol})$ in deionized water $(10 \mathrm{~mL})$ was prepared in a 50 $\mathrm{mL}$ Erlenmeyer flask. A solution of L-sodium ascorbate $(1900 \mathrm{mg}, 10 \mathrm{mmol})$ in deionized water $(15 \mathrm{~mL})$ was added to the palladium acetate solution followed by addition of a solution of trisodium citrate $(180 \mathrm{mg}, 0.60 \mathrm{mmol})$ in deionized water $(10 \mathrm{~mL})$. The combined solution was allowed to stir for $15 \mathrm{~min}$ at room temperature. During the first 10 minutes, the initial light orange color of the palladium acetate solution mixture turned black, indicating the formation of PdNP. After $15 \mathrm{~min}$, no more color changes were observed, and the solution was left to rest at room temperature open to air for $80 \mathrm{~min}$. Halloysite $(180 \mathrm{mg}, 0.60 \mathrm{mmol})$ was added to the $\mathrm{Pd}_{\mathrm{NP}}$ solution and the colloid mixture was stirred for $15 \mathrm{~min}$. The mixture was then allowed to rest at room temperature for $10 \mathrm{~min}$. The mixture was centrifuged $(6000 \mathrm{rpm})$ and the liquid was decanted away from the solid residue. The residue was washed with deionized water $(3 \times 15 \mathrm{~mL})$ and isopropyl alcohol $(2 \times 15 \mathrm{~mL})$. The resultant powder was dried at room temperature for $24 \mathrm{~h}$ in a desiccator $\left(\mathrm{CaSO}_{4}\right)$ to afford 25\% Pd@Hal as a gray powder (310 mg). 


\section{General Procedure: Suzuki-Miyaura cross-coupling reaction}

To a $50 \mathrm{~mL}$ round bottom flask equipped with a magnetic stir bar and a nitrogen inlet balloon, the aryl halide (1.0, mmol 1.2 equiv.) and the aryl boronic acid ( $1.2 \mathrm{mmol} 1.2$ equiv.) was added. The reaction mixture was flushed with nitrogen followed by the addition of $n$-propanol $(10.0 \mathrm{~mL})$ via syringe. The reaction mixture was allowed to stir the for $5 \mathrm{~min}$ allowing complete dissolution of all solids. $\mathrm{Cs}_{2} \mathrm{CO}_{3}(652 \mathrm{mg}, 2.0 \mathrm{mmol})$ was dissolved in $(2.0 \mathrm{~mL})$ of DI water, then added to the reaction mixture via syringe. The $4 \% \mathrm{Pd@Hal} \mathrm{(5 \%} \mathrm{wt/wt)} \mathrm{catalyst} \mathrm{was} \mathrm{dissolved} \mathrm{in}(2.0 \mathrm{~mL})$ of DI water; the solution was sonicated $(10 \mathrm{~min})$ to ensure dispersion, then added to the reaction mixture via syringe. The reaction flask was sonicated for 10 mins then allowed to stir at room temperature. The reaction progress was monitored by TLC. Typically, after $1 \mathrm{~h}$ the catalyst was recovered by vacuum filtration. The Pd@Hal was rinsed with ethyl acetate $(20 \mathrm{~mL})$ followed by DI water $(20 \mathrm{~mL})$ and dried in a desiccator $\left(\mathrm{CaSO}_{4}\right)$. The diluted reaction mixture was transferred to a separatory funnel and the organic phase was removed and filtered through a $2 \mathrm{~cm}$ bed of silica gel. The silica gel was rinsed with several portions of hexanes:ethyl acetate (9:1). The organic portions were combined and the solvent was removed under vacuum to afford 6. Purity was established by TLC and NMR. All products are known compounds unless otherwise indicated and spectral data were identical to those reported in the literature.

\section{Large-scale procedure: Suzuki-Miyarua cross-coupling reaction}

To a $200 \mathrm{~mL}$ round bottom flask equipped with a magnetic stir bar and a nitrogen inlet balloon, the 4-bromtoluene ( $2.05 \mathrm{~g} 12.0 \mathrm{mmol}, 1.0$ equiv.) and the 4-methoxyphenylboronic acid ( $1.85 \mathrm{~g}$, $12.2 \mathrm{mmol} 1.2$ equiv.) was added. The reaction mixture was flushed with nitrogen followed by the addition of 1-propanol $(100 \mathrm{~mL})$ via syringe. The reaction mixture was stirred the for $10 \mathrm{~min}$ allowing complete dissolution of all solids. $\mathrm{Cs}_{2} \mathrm{CO}_{3}(4.20 \mathrm{~g}, 13.0 \mathrm{mmol})$ was dissolved in $(20 \mathrm{~mL})$ of DI water, then added to the reaction mixture via syringe. The Pd@Hal catalyst $(0.100 \mathrm{~g}, 5 \% \mathrm{wt} / \mathrm{wt})$ was dissolved in $(20 \mathrm{~mL})$ of DI water; the solution was sonicated (10 min) to ensure dispersion, then added to the reaction mixture via syringe. The reaction flask was sonicated for 10 mins then allowed to stir at room temperature. The reaction progress was monitored by TLC (9:1, hexanes: ethyl acetate). The reaction was determined to be complete by the consumption of the bromotoluene and the catalyst was recovered by vacuum filtration. The Pd@Hal was rinsed with ethyl acetate $(40 \mathrm{~mL})$ followed by $\mathrm{DI}$ water $(40 \mathrm{~mL})$ and dried in a desiccator $\left(\mathrm{CaSO}_{4}\right)$. The diluted reaction mixture was transferred to a separatory funnel and the organic phase was removed and filtered through a $2 \mathrm{~cm}$ bed of silica gel. The silica gel was rinsed with several portions of (9:1, hexanes: ethyl acetate). The organic portions were combined, and the solvent was removed under vacuum to afford 3. (2.29g, 98\%). Purity was established by TLC, melting point and NMR. The product 3 is a known compound and spectral data were identical to those reported in the literature. ${ }^{1,2}$ 


\section{Recycling Studies}

The Large-scale procedure was used for the Cycle 1.

Pd@Hal recovery procedure: The Pd@Hal was removed from the filter paper and added to a beaker with $\mathrm{EtOH}(20 \mathrm{~mL})$ then sonicated for 10 mins. The solid was then centrifuged down and the washed again with $(15 \mathrm{~mL})$ DI water followed by $(15 \mathrm{~mL})$ isopropyl alcohol. The solid was dried in a desiccator $\left(\mathrm{CaSO}_{4}\right)$ overnight or in an oven at $120^{\circ}$ for 20 mins before each subsequent cycle.

Cycles 2-11. Due to loss of material during the recovery procedure for each cylce, it was necessary to adjust the reagent amounts such that a consistent catalyst loading of 5\% (wt/wt) Pd@Hal/4bromotolune was maintained.

\begin{tabular}{|c|c|}
\hline Cycle & Yield, \% \\
\hline 1 & 98 \\
\hline 2 & 99 \\
\hline 3 & 99 \\
\hline 4 & 99 \\
\hline 5 & 98 \\
\hline 6 & 97 \\
\hline 7 & 98 \\
\hline 8 & 96 \\
\hline 9 & 92 \\
\hline 10 & 85 \\
\hline 11 & 52 \\
\hline
\end{tabular}


Table 1, Entry 1

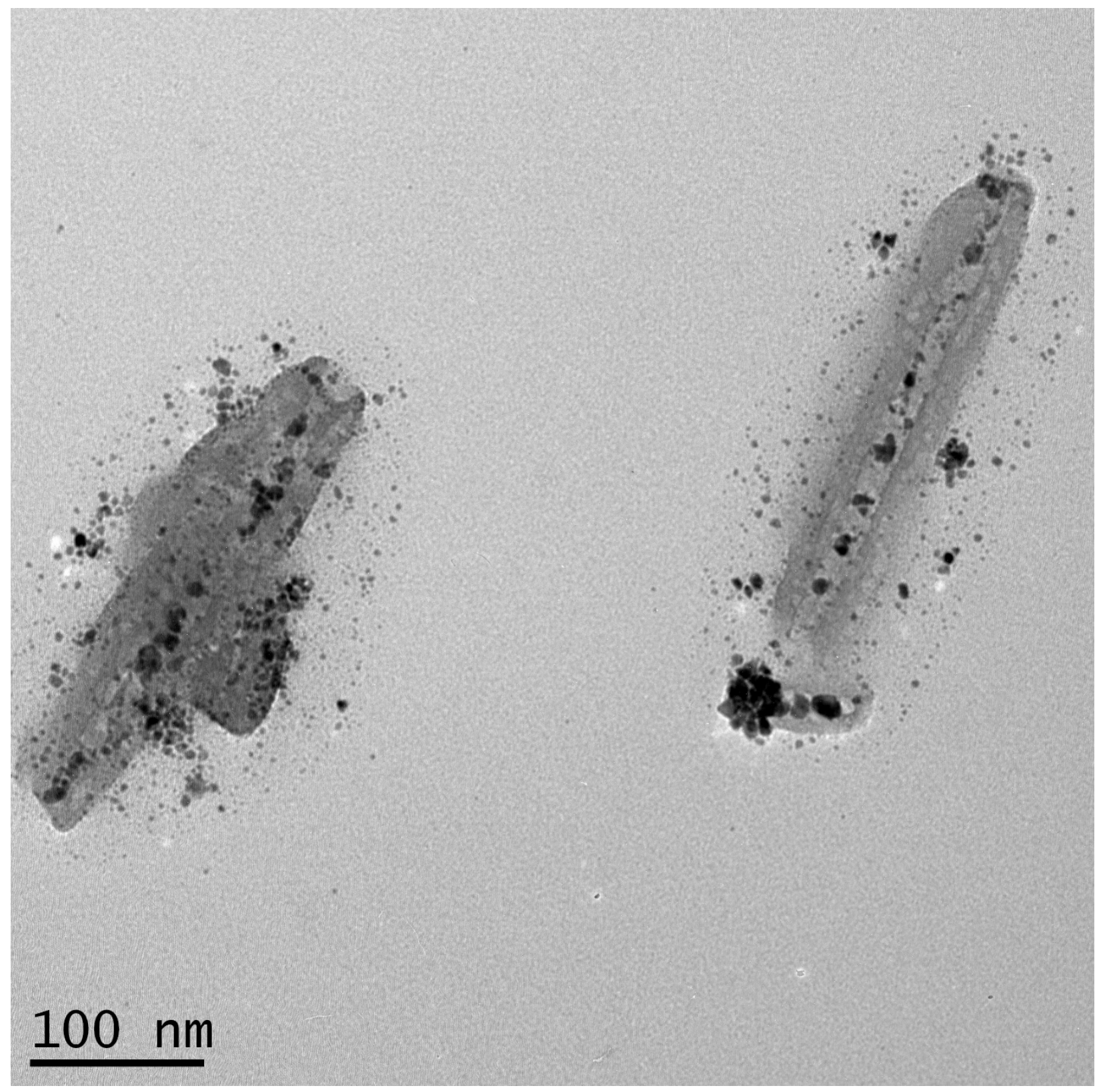


Table 1, Entry 2

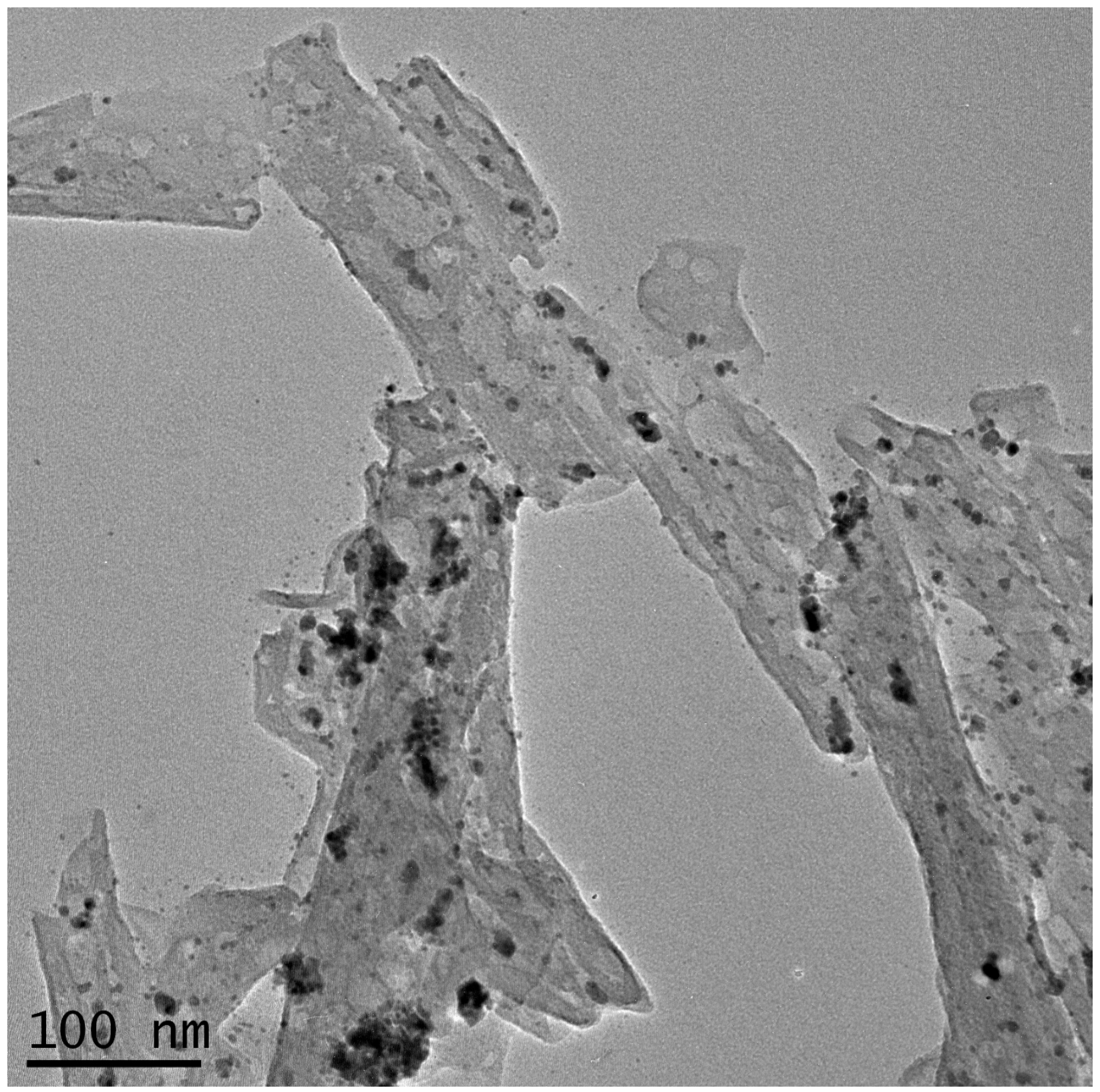


Table 1, Entry 3

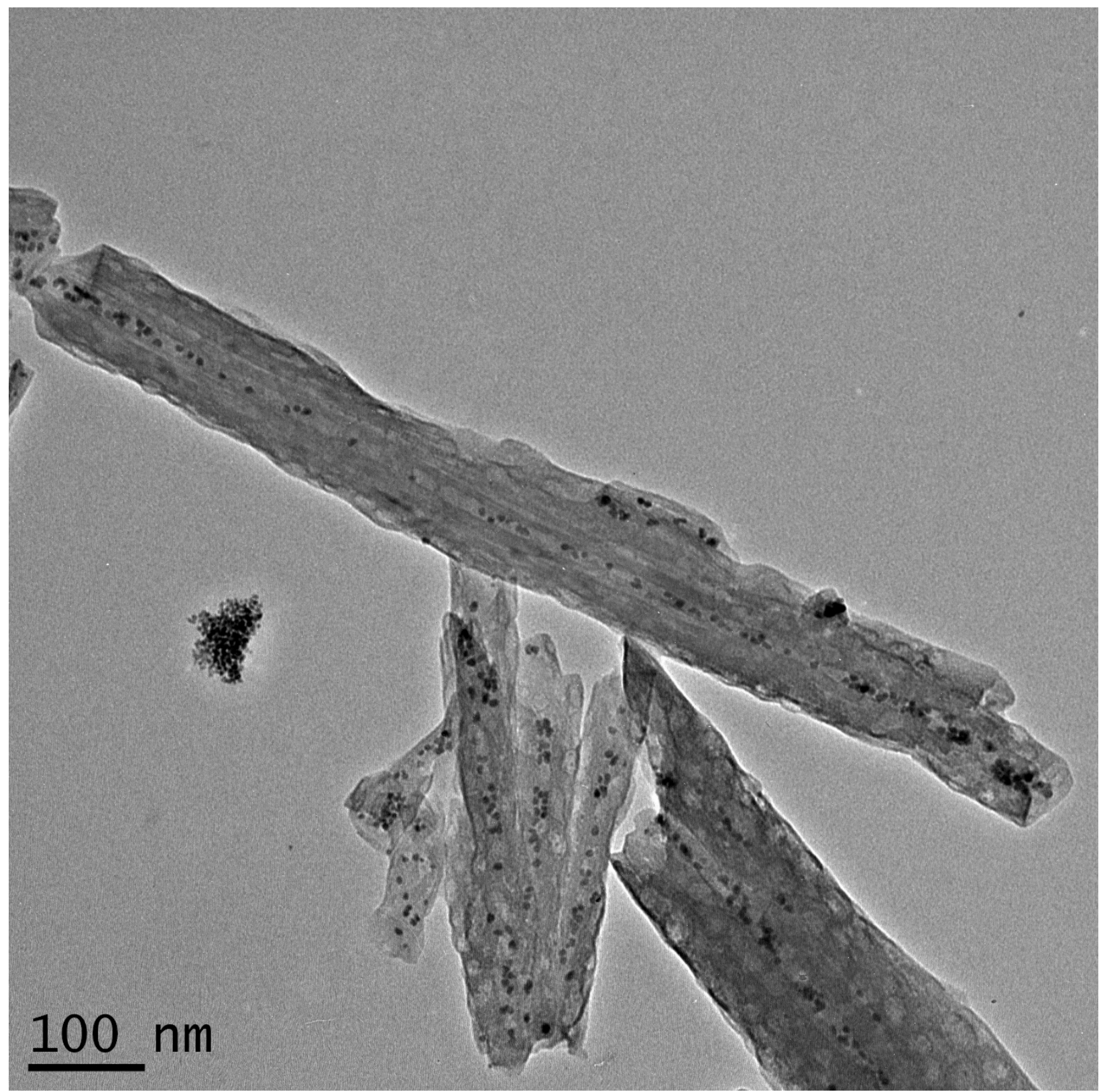


Table 1, Entry 4

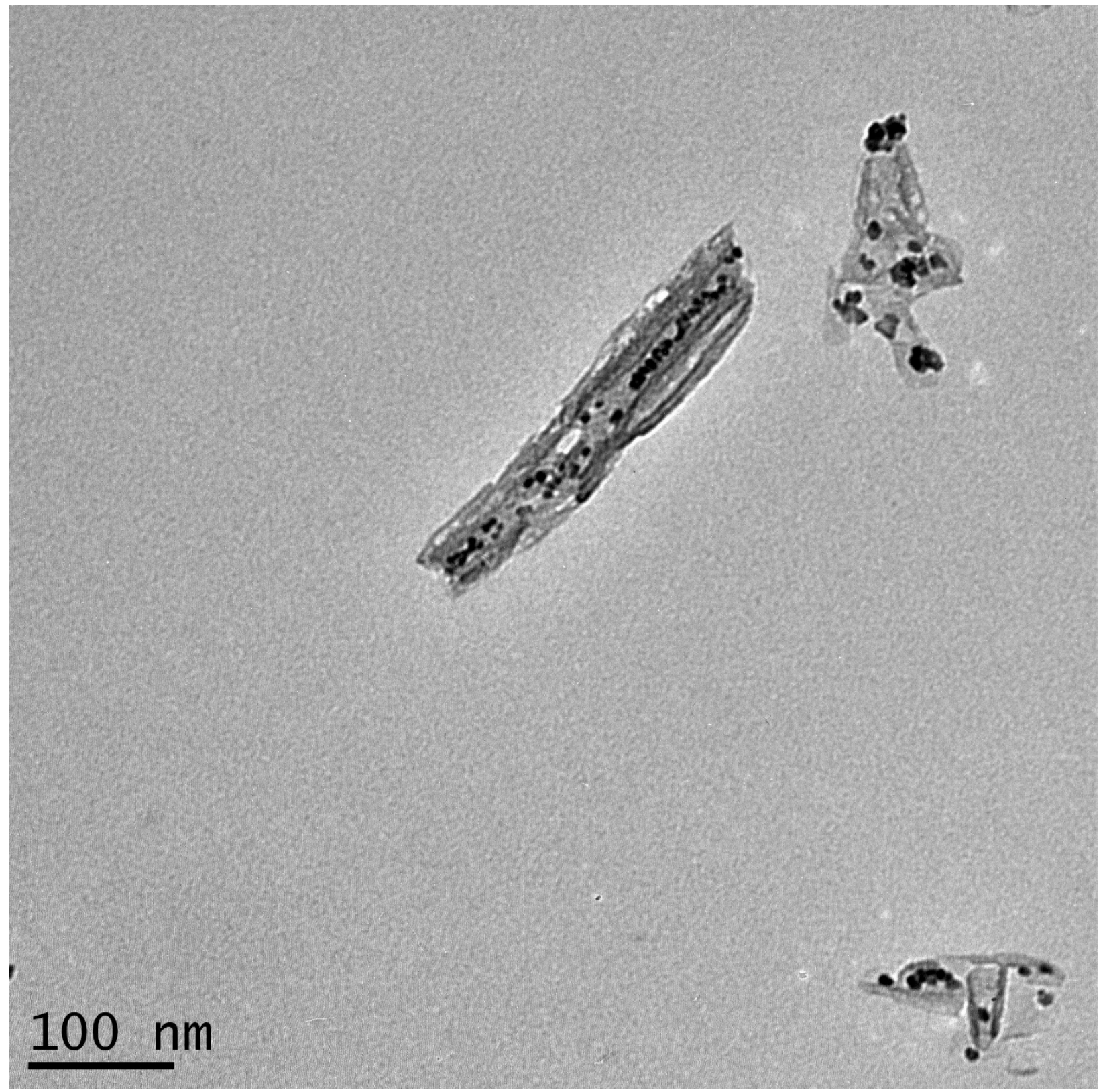


Table 1, Entry 5

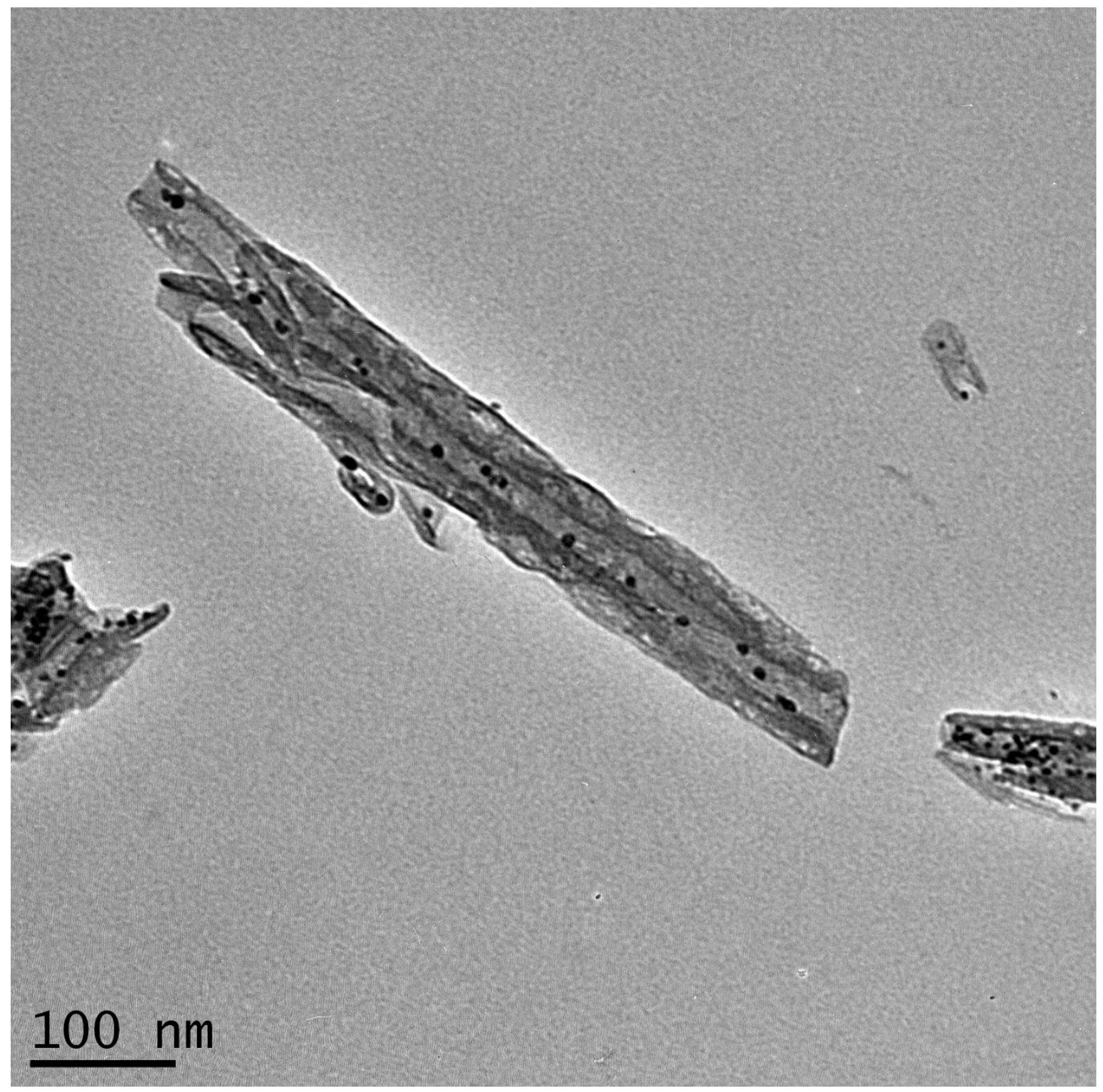


Table 1, Entry 6

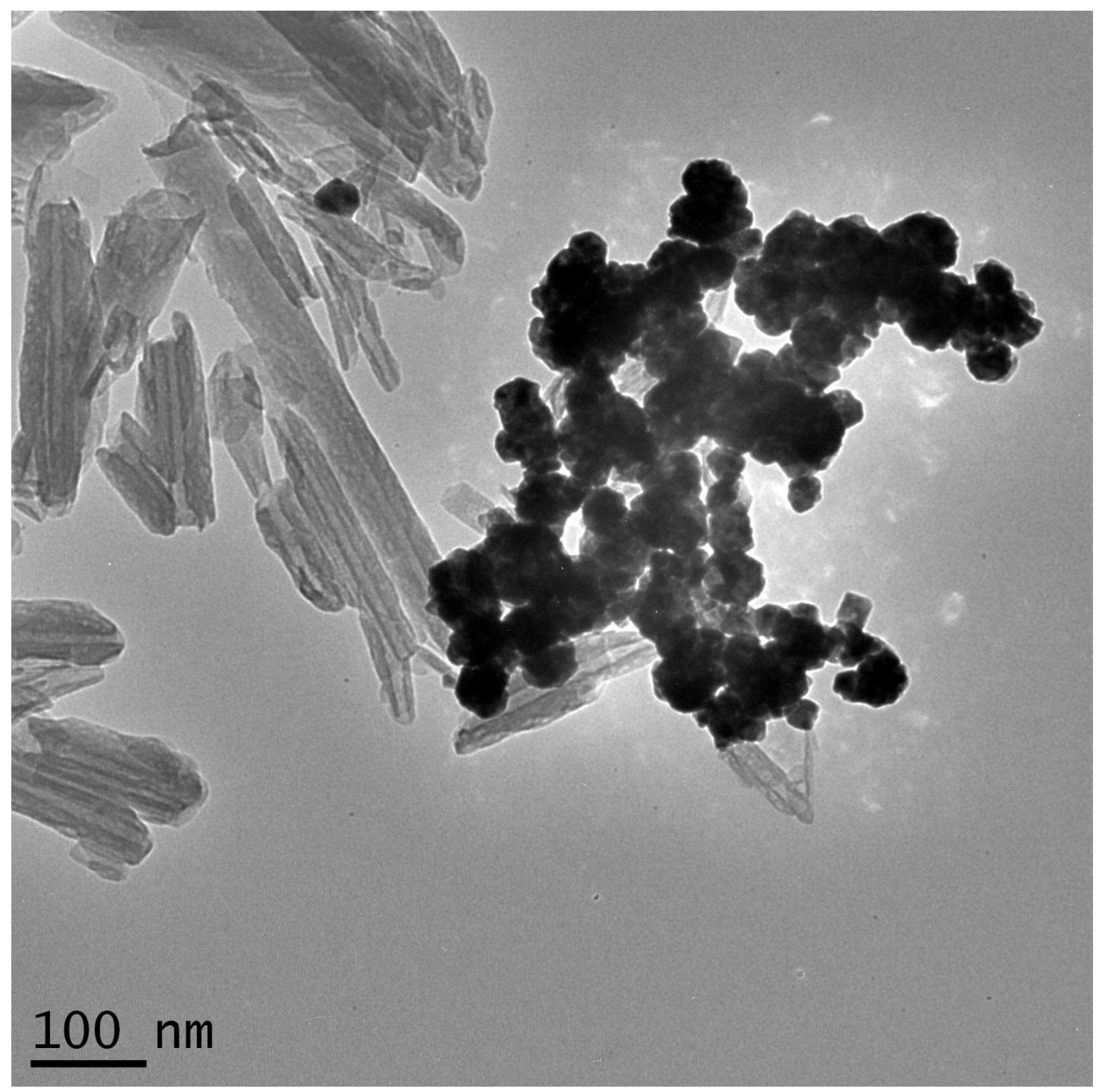


Table 1, Entry 7

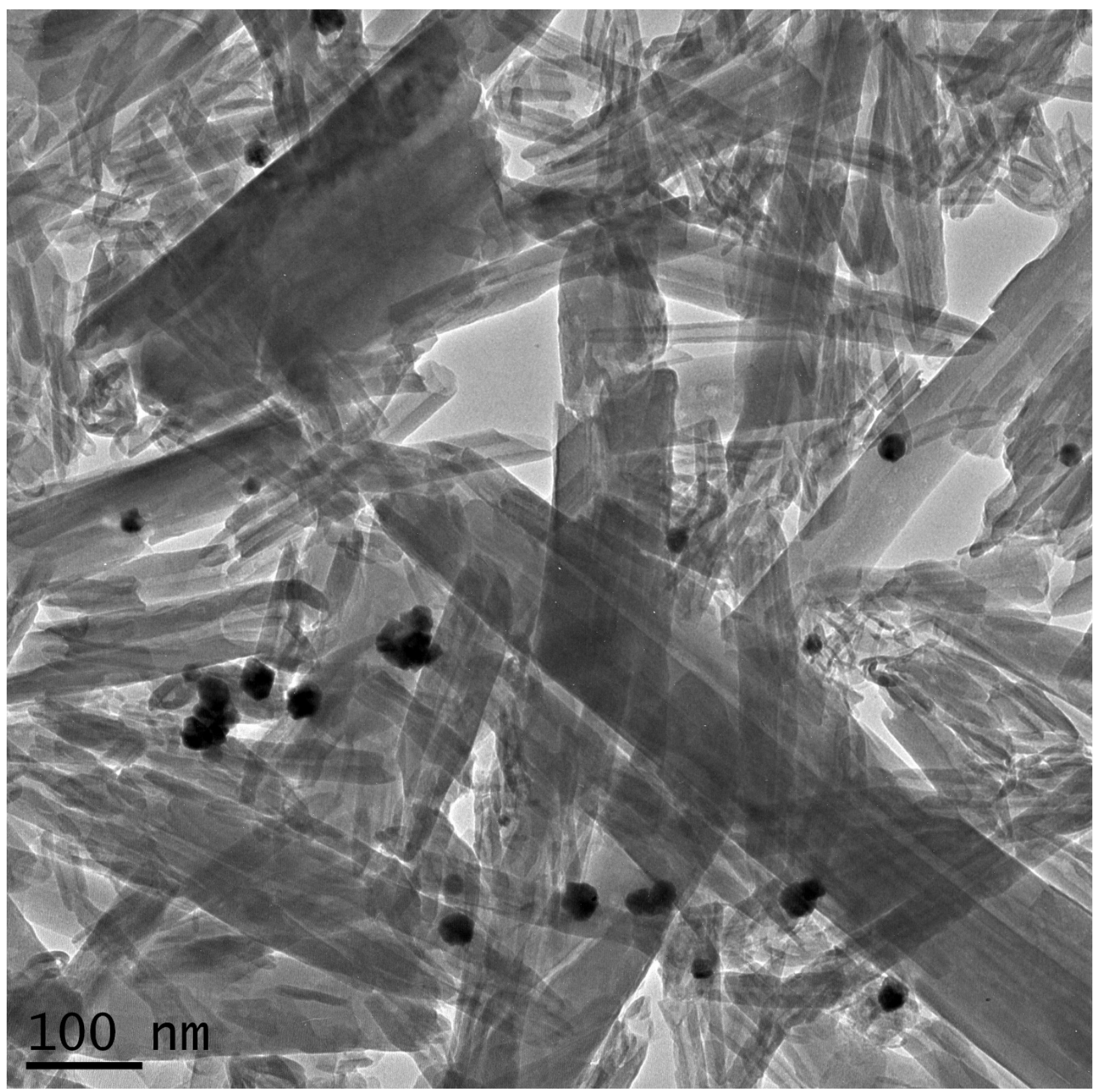


Table 1, Entry 8

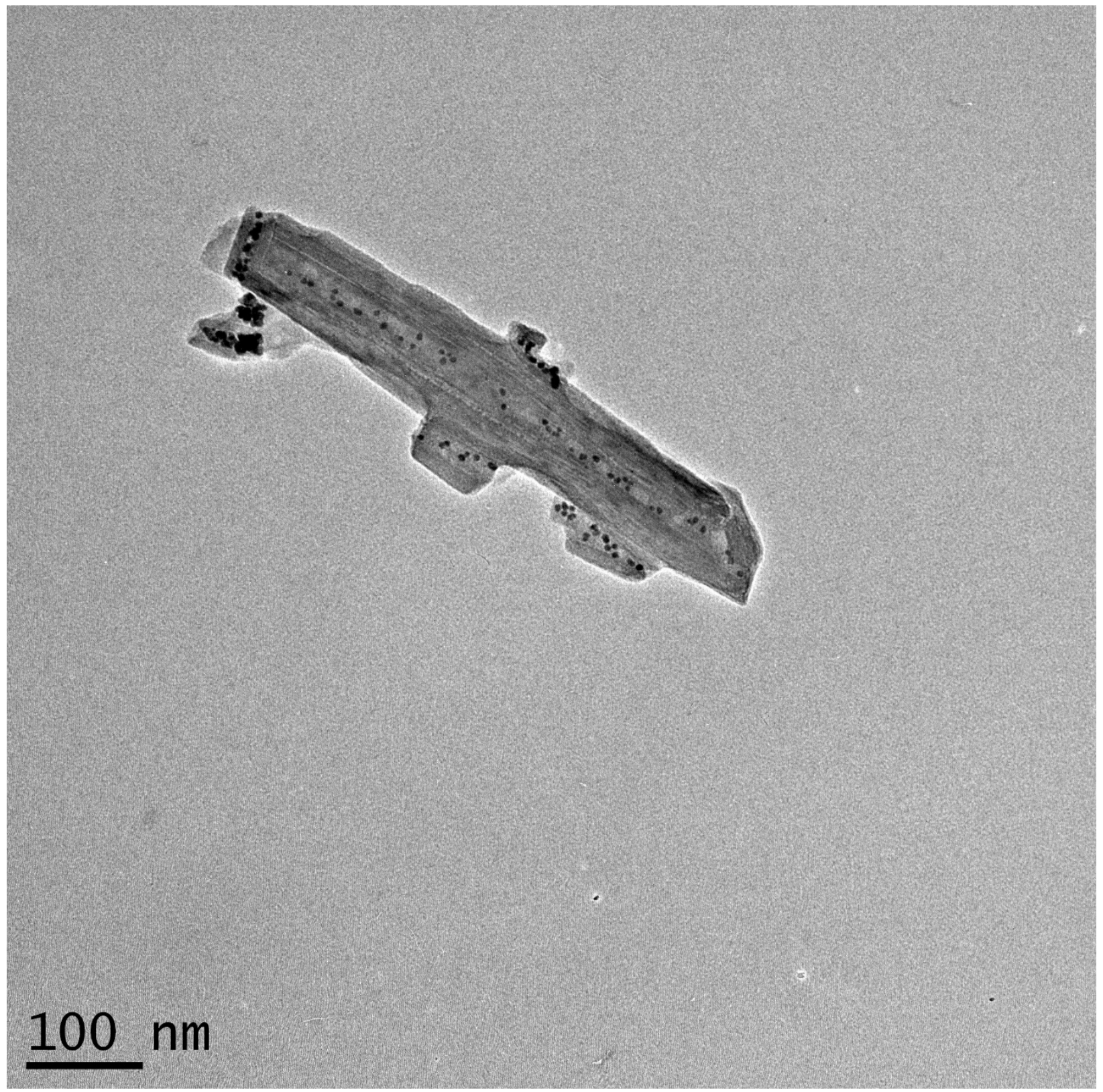


Table 1, Entry 9

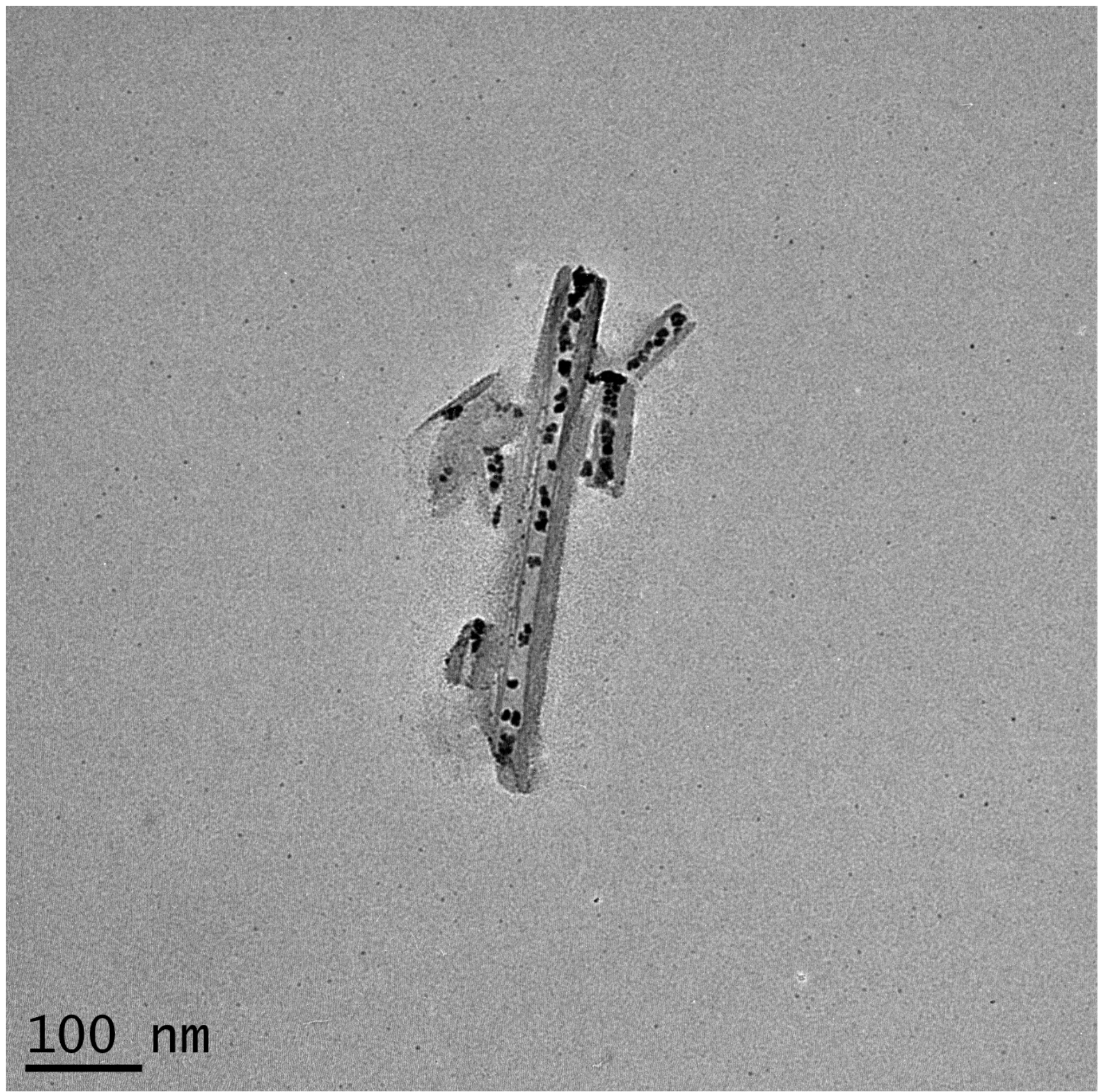


Table 1, Entry 10

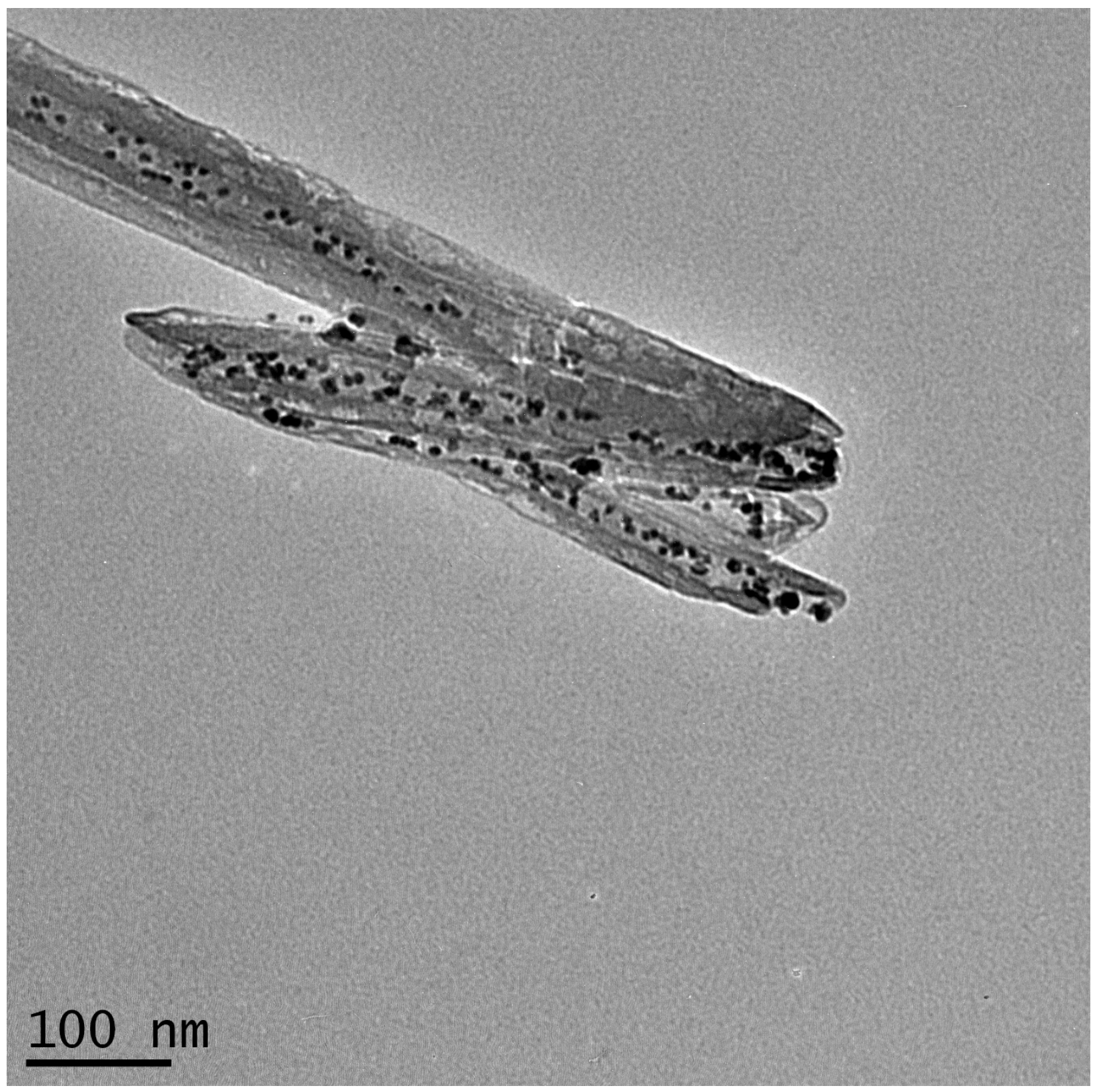




\section{Recycling Studies}

\section{Pd@Hal (Cycle 1)}

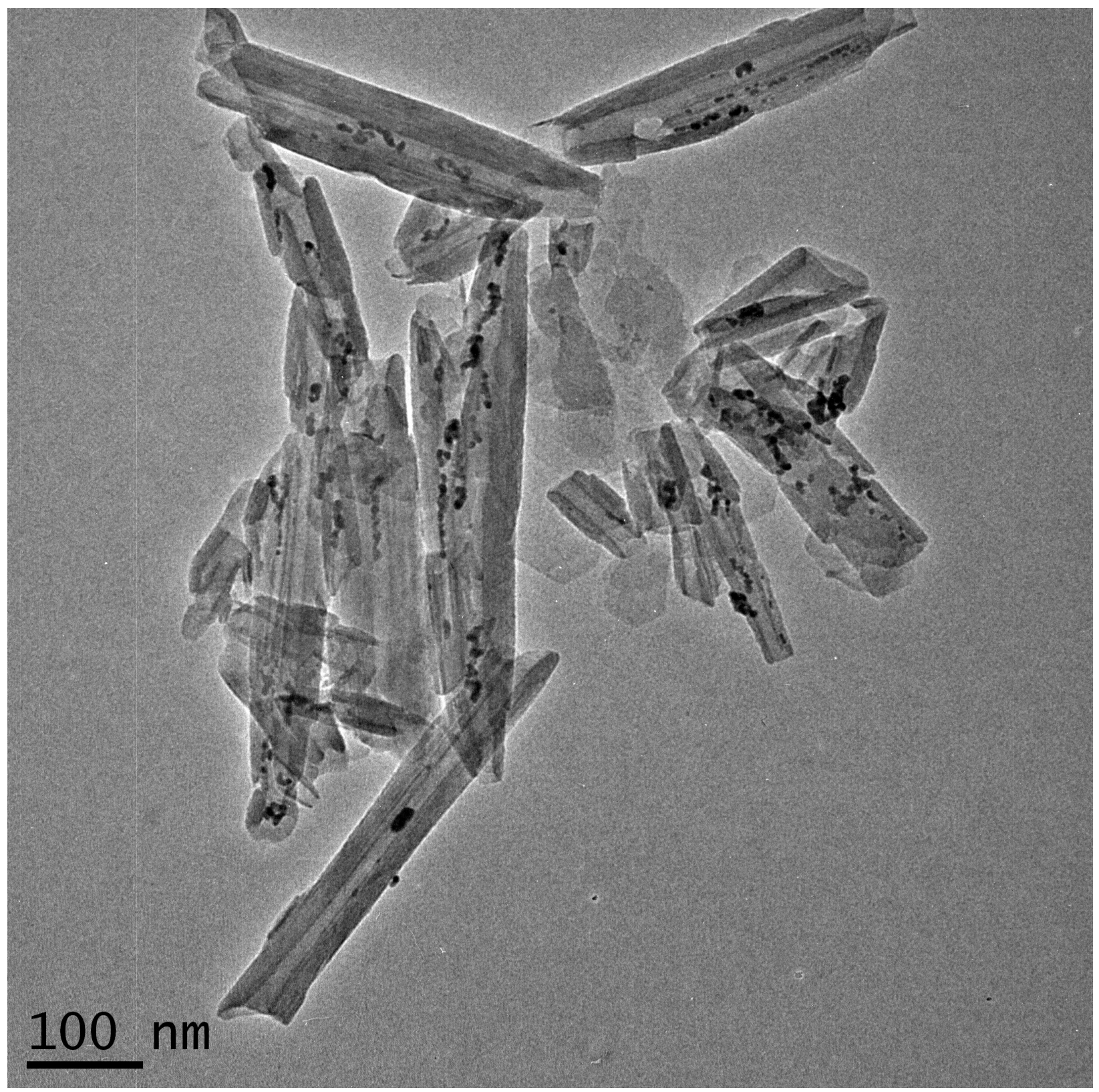


Pd@Hal (after three uses)

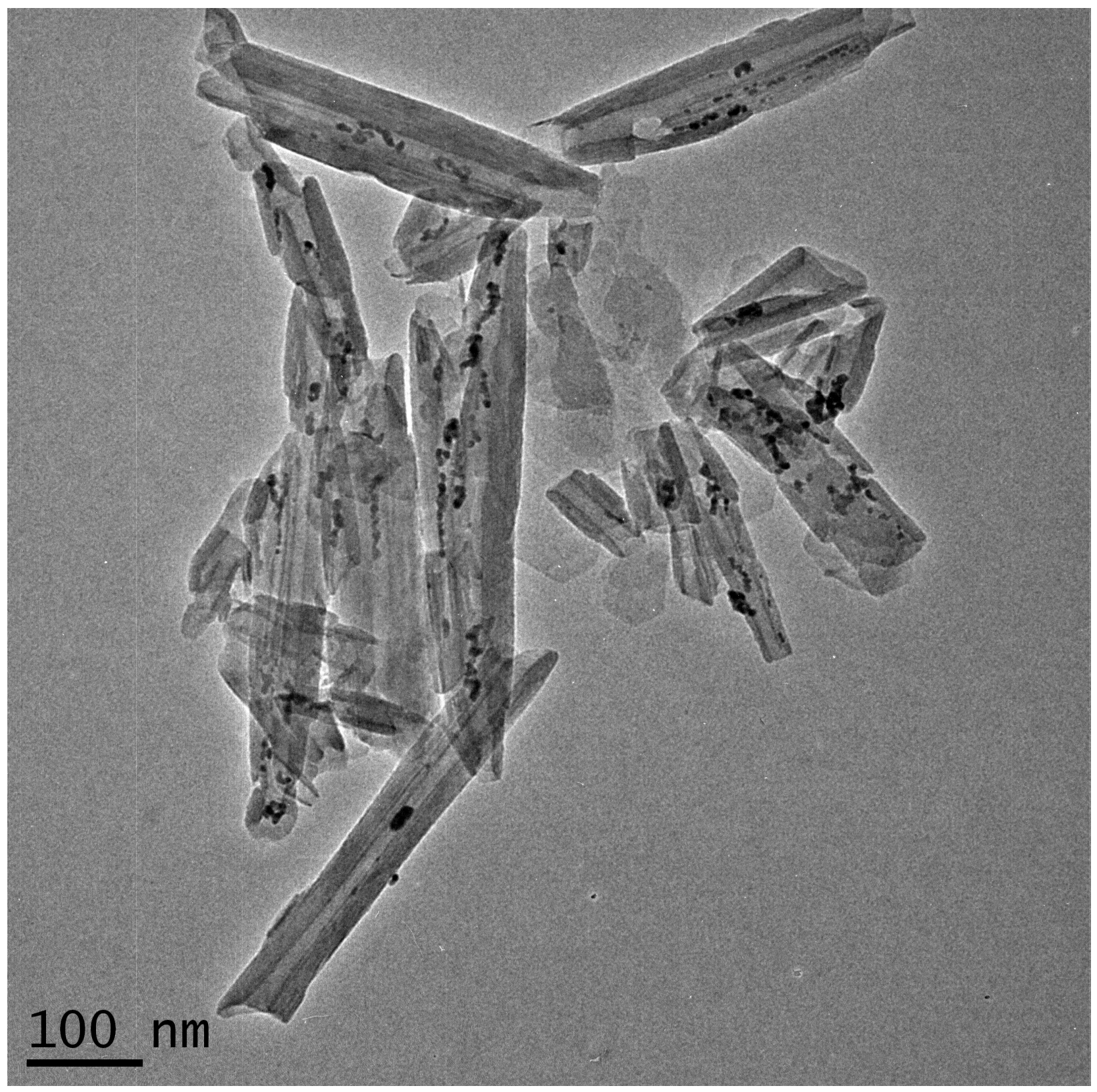




\section{Characterization of Coupling Products (6, Scheme 1)}

All the products are known compounds and the spectral data and melting points were identical to those reported in the literature. ${ }^{1} \mathrm{H}$ NMR are shown below each entry to illustrate the purity of the sample.

4-methoxy-4'-methyl-1,1'-biphenyl (6a)

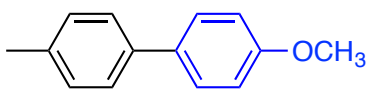

White solid (0.195 g, 98\%); mp 109-110 ${ }^{\circ} \mathrm{C}$. [Lit. mp 107.9-108. $\left.{ }^{\circ} \mathrm{C}\right]^{1}$

${ }^{1} \mathrm{H}$ NMR $\left(400 \mathrm{MHz}\right.$, DMSO- $\left.d_{6}\right) \delta: 7.55(\mathrm{~d}, \mathrm{~J}=8.8 \mathrm{~Hz}, 2 \mathrm{H}), 7.48(\mathrm{~d}, \mathrm{~J}=8.0 \mathrm{~Hz}, 2 \mathrm{H}), 7.21(\mathrm{~d}, \mathrm{~J}=7.6 \mathrm{~Hz}, 2 \mathrm{H}$, $6.98(\mathrm{~d}, \mathrm{~J}=8.4 \mathrm{~Hz}, 2 \mathrm{H}), 3.76(\mathrm{~s}, 3 \mathrm{H}), 2.30(\mathrm{~s}, 3 \mathrm{H})$. Identical to ${ }^{1} \mathrm{H}$ NMR reported in the literature. ${ }^{2}$

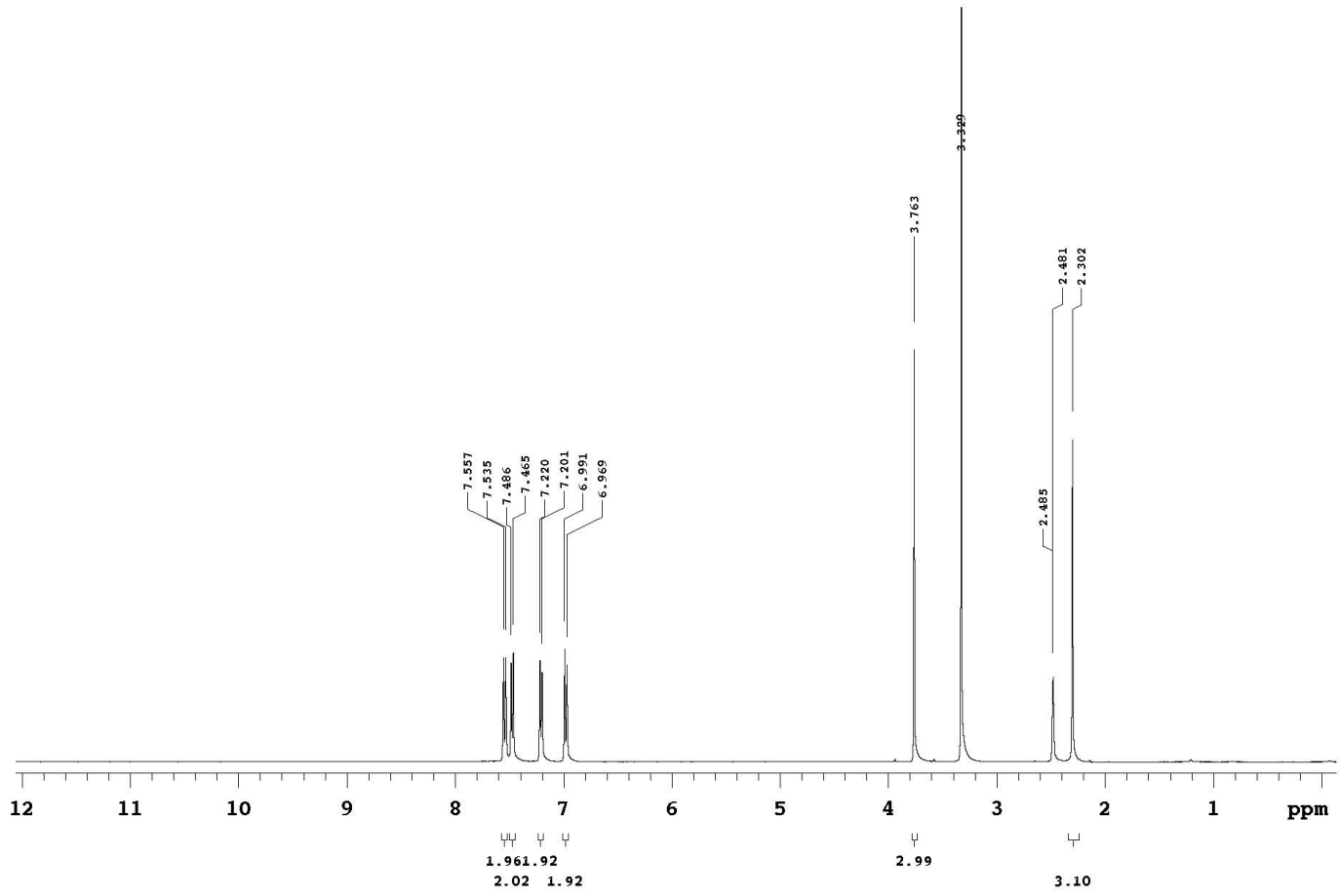


4,4'-dimethyl-1,1'-biphenyl (6b)

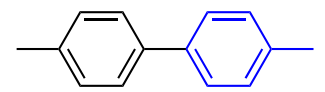

White solid (0.168 g, 92\%); mp $118-119.5^{\circ} \mathrm{C}$. [Lit. mp $\left.121.5-122.0^{\circ} \mathrm{C}\right]^{3}$

${ }^{1} \mathrm{H}$ NMR (400 MHz, DMSO- $d_{6}$ ) $\delta: 7.50(\mathrm{~d}, \mathrm{~J}=8.4 \mathrm{~Hz}, 4 \mathrm{H}), 7.23(\mathrm{~d}, \mathrm{~J}=8.0 \mathrm{~Hz}, 4 \mathrm{H}), 2.31(\mathrm{~s}, 6 \mathrm{H})$. Identical to ${ }^{1} \mathrm{H}$ NMR reported in the literature. ${ }^{3}$

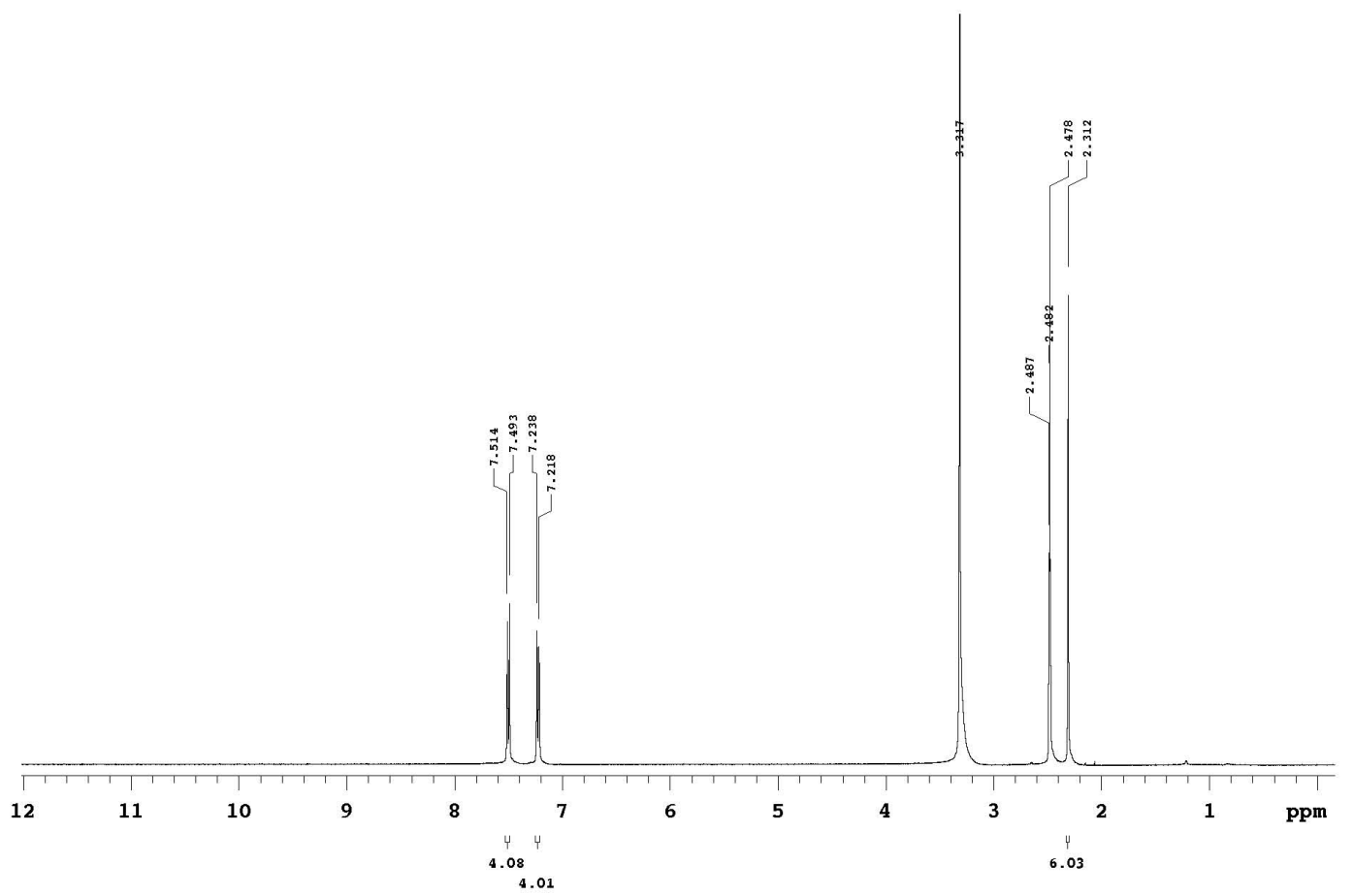


4-methoxy-1,1'-biphenyl (6c/6d)

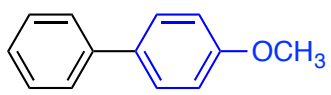

White solid (0.178 g/0.180, 97\%/98\%); mp 89.2-91.1 ${ }^{\circ} \mathrm{C}$. [Lit. $\left.\mathrm{mp} 85.3-85.7^{\circ} \mathrm{C}\right]^{1 .}$

${ }^{1} \mathrm{H}$ NMR $\left(400 \mathrm{MHz}, \mathrm{DMSO}-d_{6}\right) \delta: 7.58(\mathrm{dd}, \mathrm{J}=8.4,1.6 \mathrm{~Hz}, 4 \mathrm{H}), 7.41(\mathrm{t}, \mathrm{J}=7.6 \mathrm{~Hz}, 2 \mathrm{H}), 7.29(\mathrm{t}, \mathrm{J}=7.2 \mathrm{~Hz}$, $1 \mathrm{H}), 7.01(\mathrm{~d}, \mathrm{~J}=8.8 \mathrm{~Hz}, 2 \mathrm{H}), 3.78(\mathrm{~s}, 3 \mathrm{H})$. Identical to ${ }^{1} \mathrm{H}$ NMR reported in the literature. ${ }^{4}$

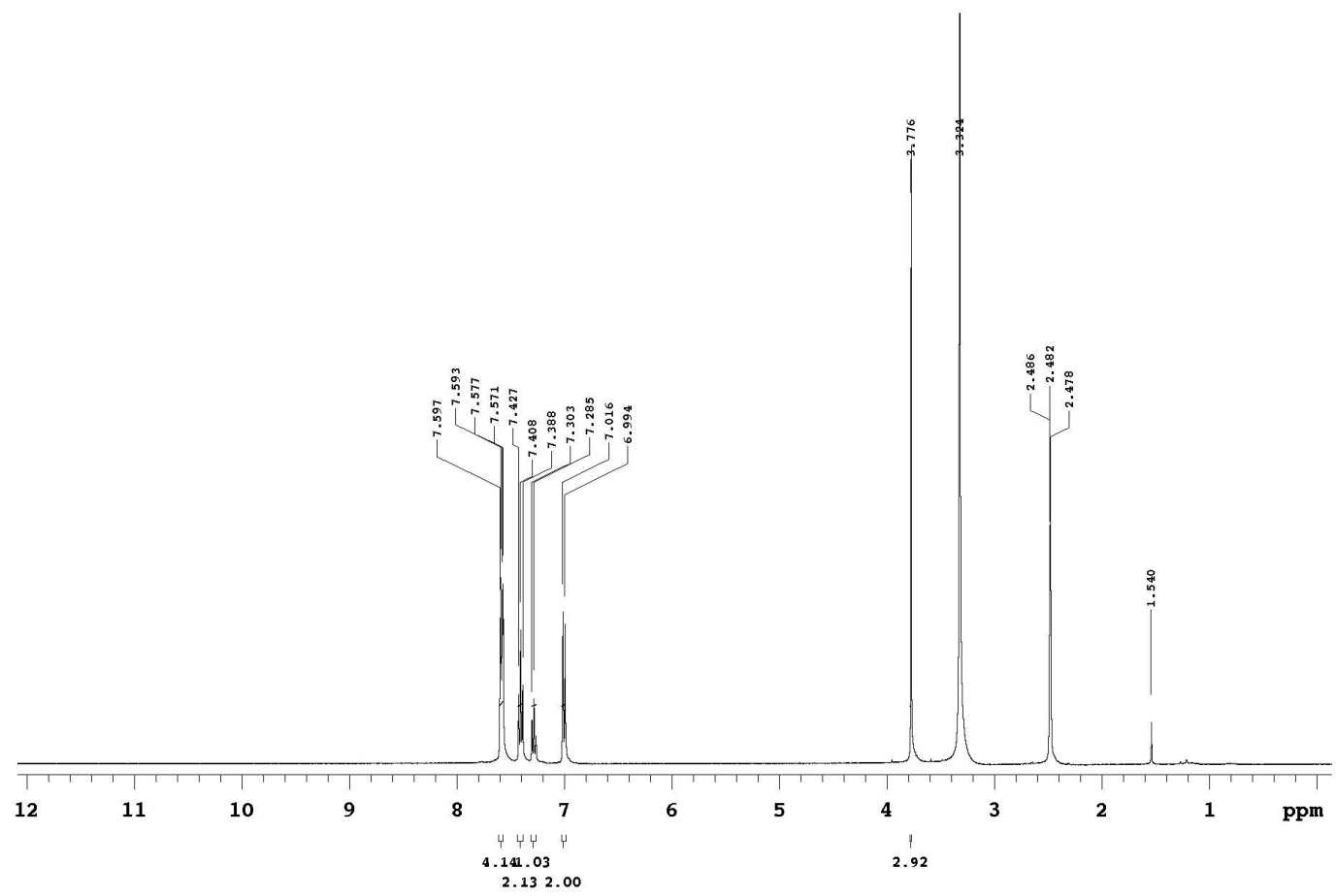


4-fluoro-4'-methyl-1,1'-biphenyl (6e)

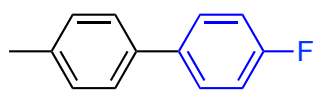

White solid (0.178 g, 96\%); $m p$ 76.5-77. $1^{\circ} \mathrm{C}$ \{Lit. $\mathrm{mp} \mathrm{78-79]^{5 }}$

${ }^{1} \mathrm{H}$ NMR $\left(400 \mathrm{MHz}, \mathrm{DMSO}-\mathrm{d}_{6}\right) \delta: 7.65$ (dd, J = 8.8, 5,6 Hz, 2H), $7.51(\mathrm{~d}, \mathrm{~J}=7.6 \mathrm{~Hz}, 2 \mathrm{H}), 7.27-7.22(\mathrm{~m}, 4 \mathrm{H})$, $2.48(\mathrm{~s}, 3 \mathrm{H})$. Identical to ${ }^{1} \mathrm{H}$ NMR reported in the literature. ${ }^{6}$

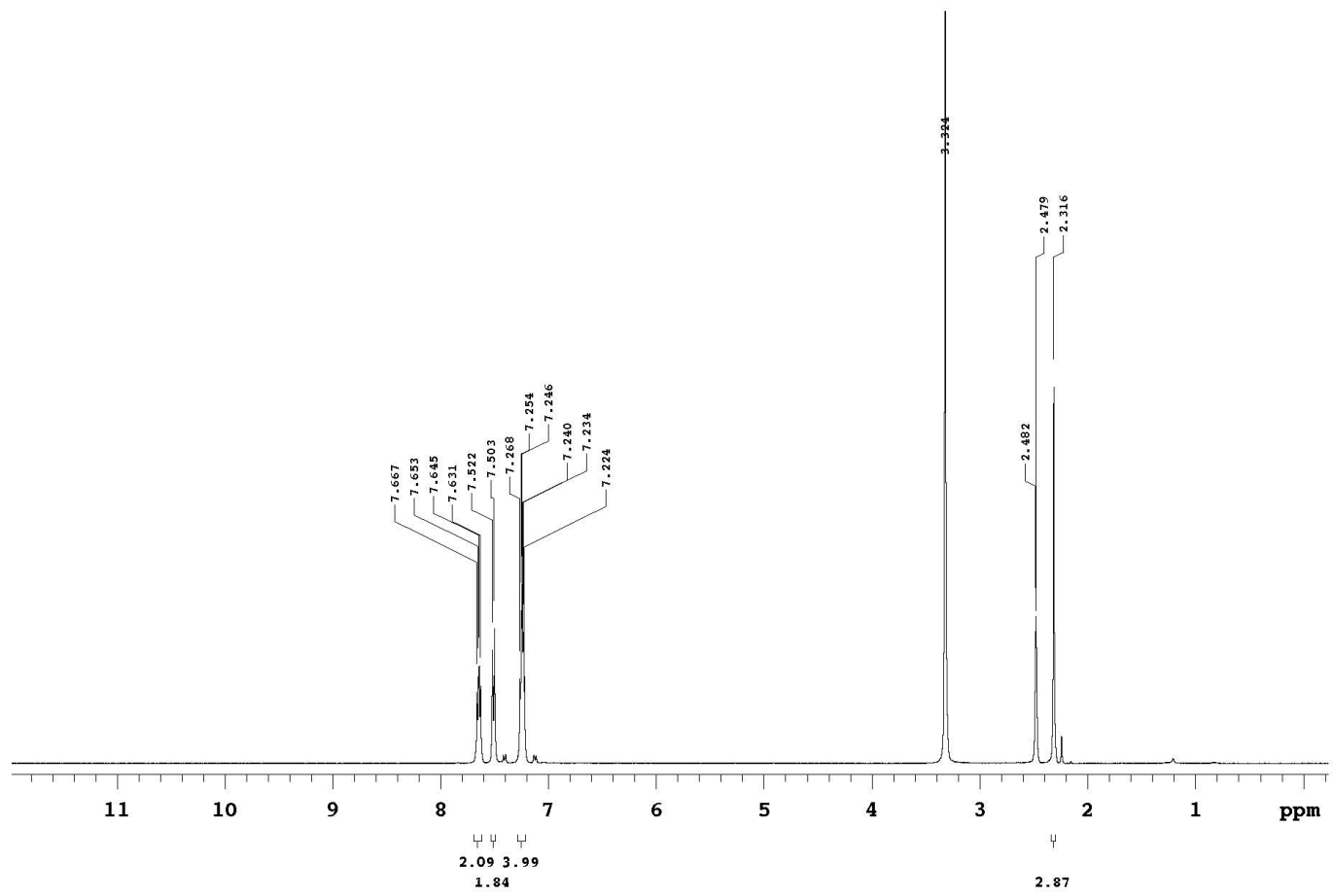


4-fluoro-4'-methoxy-1,1'-biphenyl (6f)

$\longrightarrow \mathrm{OCH}_{3}$

White solid (0.198 g, 98\%); $\mathrm{mp} 88.6-89.5^{\circ} \mathrm{C}$ [Lit. mp: $\left.87.3-87.8^{\circ} \mathrm{C}\right]^{1}$

${ }^{1} \mathrm{H}$ NMR $\left(400 \mathrm{MHz}, \mathrm{DMSO}-\mathrm{d}_{6}\right) \delta: 7.64-7.60(\mathrm{~m}, 2 \mathrm{H}), 7.56(\mathrm{~d}, \mathrm{~J}=8.8 \mathrm{~Hz}, 2 \mathrm{H}), 7.23(\mathrm{t}, \mathrm{J}=8.8 \mathrm{~Hz}, 2 \mathrm{H}), 7.00$ $(\mathrm{d}, \mathrm{J}=8.8 \mathrm{~Hz}, 2 \mathrm{H}), 3.77(\mathrm{~s}, 3 \mathrm{H})$. Identical to ${ }^{1} \mathrm{H}$ NMR reported in the literature. ${ }^{7}$

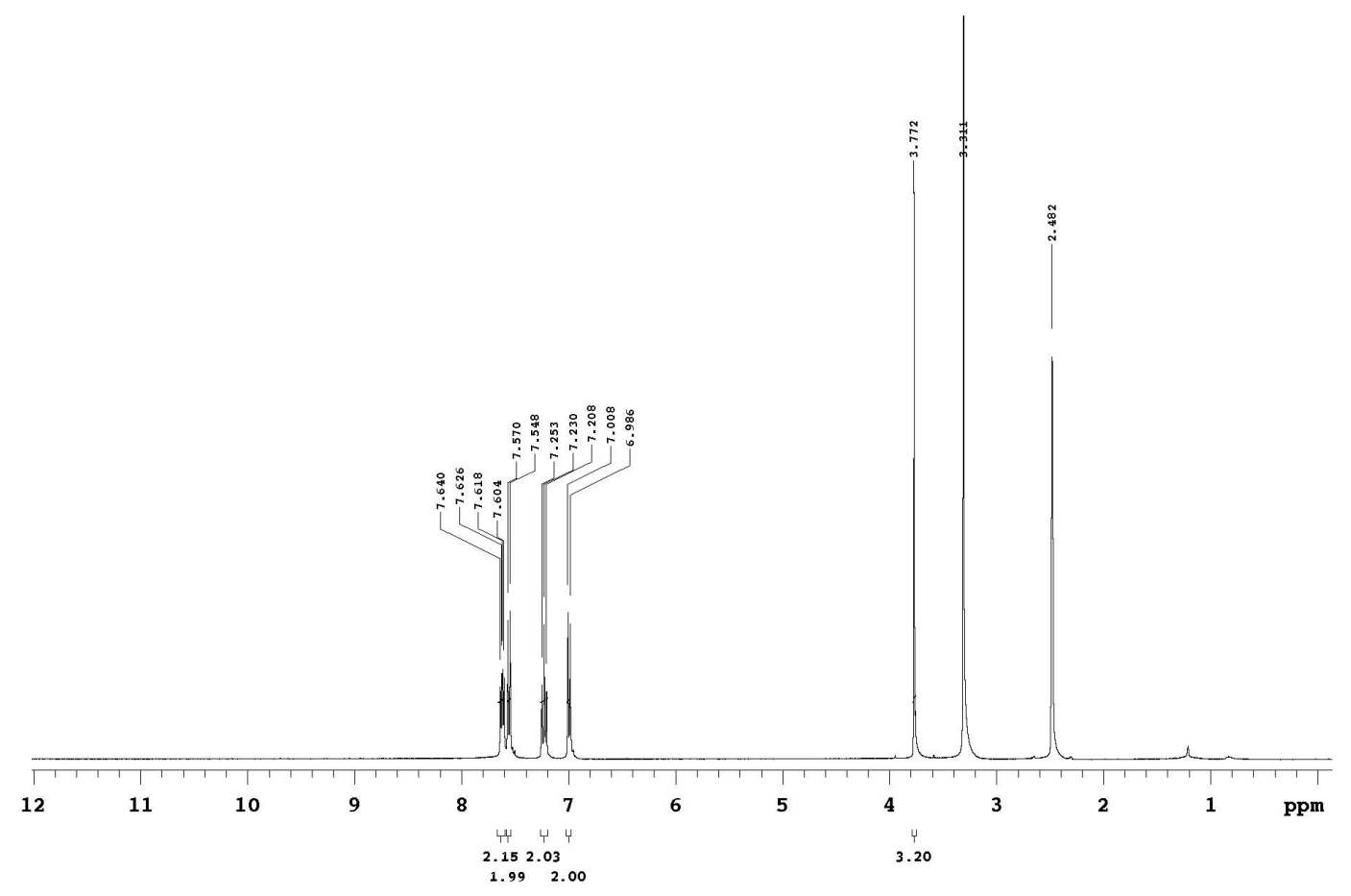


2-phenyl-naphthalene (6g)

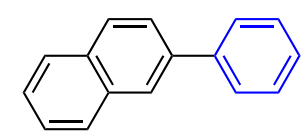

White solid ( $0.197 \mathrm{~g}, 96 \%) ; \mathrm{mp} 102.5-103.8^{\circ} \mathrm{C}\left[\right.$ Lit. $\left.\mathrm{mp} 101^{\circ} \mathrm{C}\right] .^{8}$

${ }^{1} \mathrm{H}$ NMR $\left(300 \mathrm{MHz}\right.$, DMSO- $\left.d_{6}\right) \delta: 8.19(\mathrm{~d}, \mathrm{~J}=7.2 \mathrm{~Hz}, 1 \mathrm{H})$, 7.99-7.91 $(\mathrm{m}, 3 \mathrm{H})$, 7.88-7.77 $(\mathrm{m}, 3 \mathrm{H})$, 7.64-7.49 $(\mathrm{m}, 4 \mathrm{H}), 7.40-7.35(\mathrm{~m}, 1 \mathrm{H})$. Identical to ${ }^{1} \mathrm{H}$ NMR reported in the literature. ${ }^{9}$

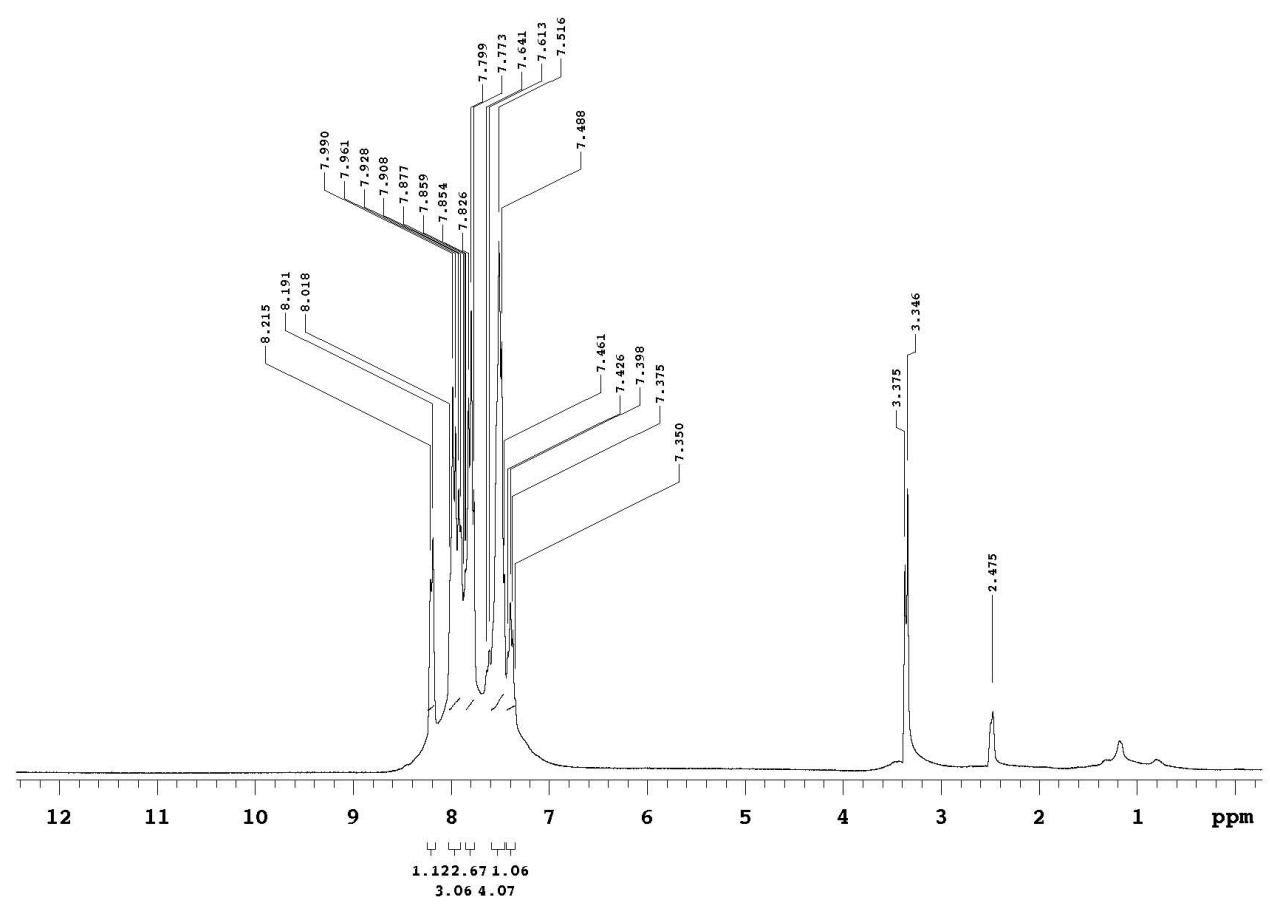




\section{2-(4-fluorophenyl)naphthalene (6h)}

White solid (0.202 g, 91\%); mp 101.1-103.2

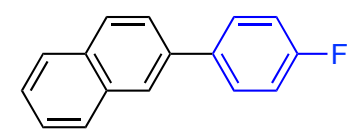

${ }^{1} \mathrm{H}$ NMR $\left(300 \mathrm{MHz}, \mathrm{DMSO}-d_{6}\right) \delta: 8.19(\mathrm{~s}, 1 \mathrm{H}), 8.00-7.92(\mathrm{~m}, 3 \mathrm{H}), 7.86-7.80(\mathrm{~m}, 3 \mathrm{H}), 7.53-7.50(\mathrm{~m}, 2 \mathrm{H})$, $7.39(\mathrm{t}, \mathrm{J}=8.7 \mathrm{~Hz}, 2 \mathrm{H})$. Identical to ${ }^{1} \mathrm{H}$ NMR reported in the literature. ${ }^{9}$

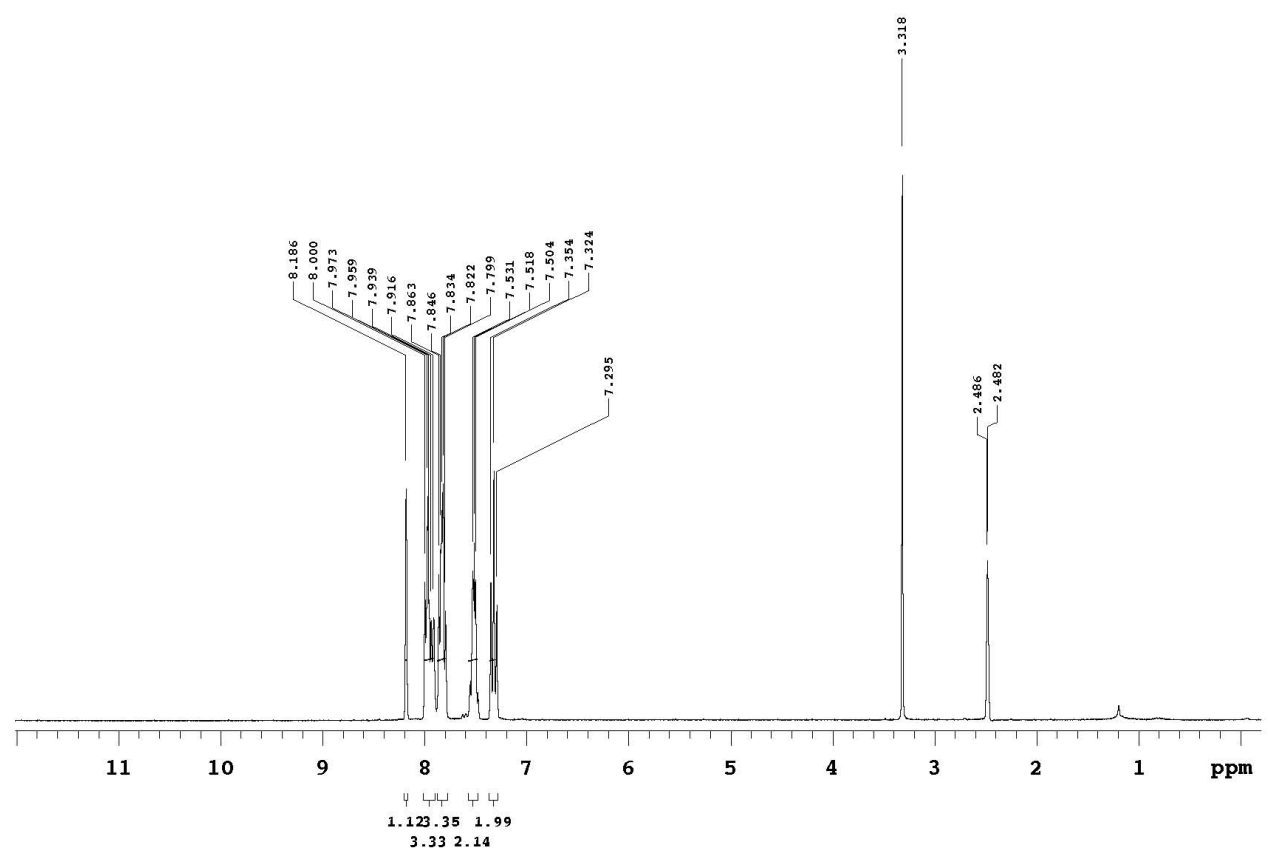




\section{4'-methyl-biphenyl-4-carbonitrile (6i)}

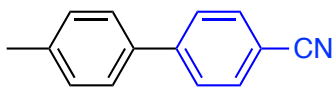

White solid (0.182 g, 94\%); mp $110.6-111.8^{\circ} \mathrm{C}\left[\text { Lit. } \mathrm{mp} 110-111^{\circ} \mathrm{C}\right]^{10}$

${ }^{1} \mathrm{H}$ NMR $\left(400 \mathrm{MHz}, \mathrm{DMSO}-d_{6}\right) \delta: 7.89(\mathrm{~d}, \mathrm{~J}=8.4 \mathrm{~Hz}, 2 \mathrm{H}), 7.84(\mathrm{~d}, \mathrm{~J}=8.4 \mathrm{~Hz}, 2 \mathrm{H}), 7.64(\mathrm{~d}, \mathrm{~J}=8.0 \mathrm{~Hz}, 2 \mathrm{H})$, $7.31(\mathrm{~d}, \mathrm{~J}=7.6 \mathrm{~Hz}, 2 \mathrm{H}), 2.34(\mathrm{~s}, 3 \mathrm{H})$. Identical to ${ }^{1} \mathrm{H}$ NMR reported in the literature. ${ }^{11}$

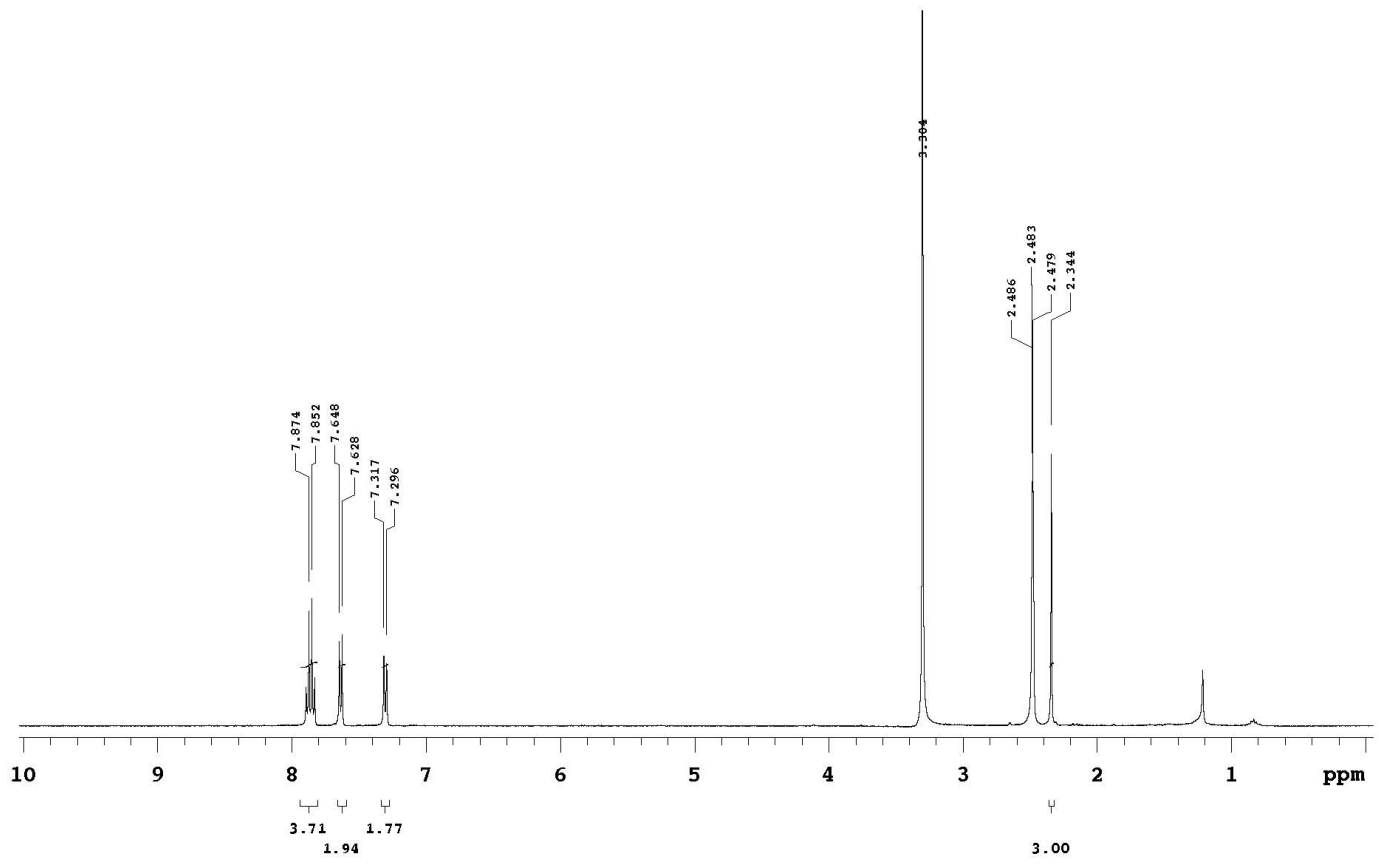


1-(4'-Methyl[1,1'-biphenyl]-4-yl)ethanone (6j)

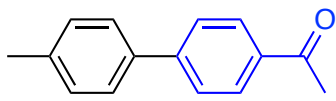

White solid (0.194 g, 92\%); mp $120.3-122.1^{\circ} \mathrm{C}$ [Lit. mp $\left.122-124{ }^{\circ} \mathrm{C}\right]^{12}$

${ }^{1} \mathrm{H} \mathrm{NMR}\left(300 \mathrm{MHz}, \mathrm{DMSO}-\mathrm{d}_{6}\right) \delta: 8.00(\mathrm{~d}, \mathrm{~J}=6.0 \mathrm{~Hz}, 2 \mathrm{H}), 7.78(\mathrm{~d}, \mathrm{~J}=6.0 \mathrm{~Hz}, 2 \mathrm{H}), 7.63(\mathrm{~d}, \mathrm{~J}=6.0 \mathrm{~Hz}, 2 \mathrm{H})$, $7.29(\mathrm{~d}, \mathrm{~J}=5.7 \mathrm{~Hz}, 2 \mathrm{H}), 2.58(\mathrm{~s}, 3 \mathrm{H}), 2.34(\mathrm{~s}, 3 \mathrm{H})$. Identical to ${ }^{1} \mathrm{H}$ NMR reported in the literature. ${ }^{12}$

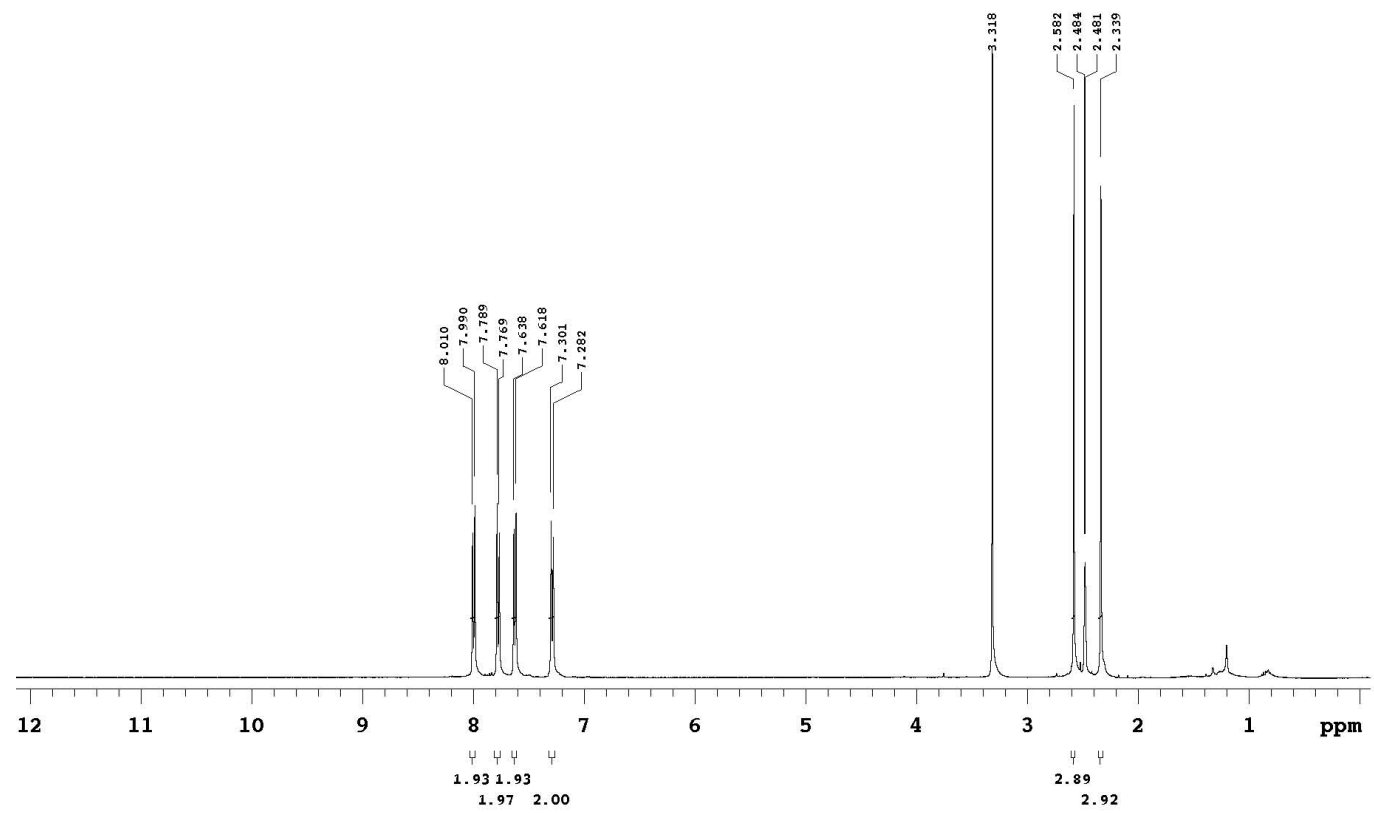


3-(4-methoxyphenyl)pyridine (6k)

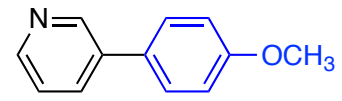

Light yellow solid $(0.178 \mathrm{~g}, 97 \%) ; \mathrm{mp} 61.5-63.1^{\circ} \mathrm{C}\left[\right.$ Lit. $\left.\mathrm{mp} 62-63^{\circ} \mathrm{C}\right] .^{13}$

${ }^{1} \mathrm{H} \mathrm{NMR}\left(400 \mathrm{MHz}, \mathrm{DMSO}-\mathrm{d}_{6}\right) \delta: 8.83(\mathrm{~s}, 1 \mathrm{H}), 8.49(\mathrm{~d}, \mathrm{~J}=4.0 \mathrm{~Hz}, 1 \mathrm{H}), 8.00(\mathrm{~d}, \mathrm{~J}=8.4 \mathrm{~Hz}, 1 \mathrm{H}), 7.66(\mathrm{~d}, \mathrm{~J}=$ $8.4 \mathrm{~Hz}, 2 \mathrm{H}), 7.43(\mathrm{~m}, 1 \mathrm{H}), 7.04(\mathrm{~d}, \mathrm{~J}=8.4 \mathrm{~Hz}, 2 \mathrm{H}), 3.79(\mathrm{~s}, 3 \mathrm{H})$. Identical to ${ }^{1} \mathrm{H}$ NMR reported in the literature. ${ }^{13}$

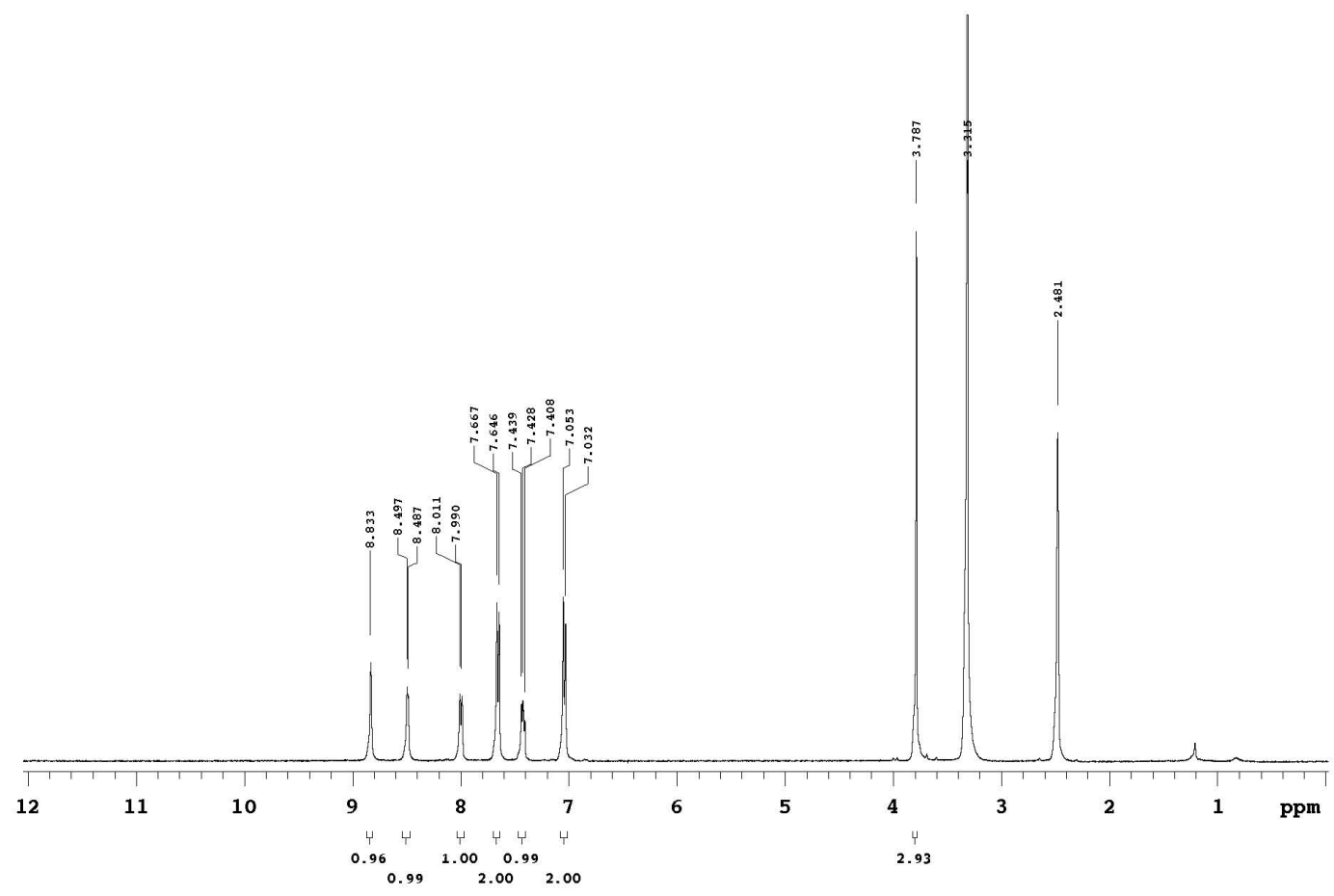


3-chloro-4'-methoxy-4-methyl-1,1'-biphenyl (6I)

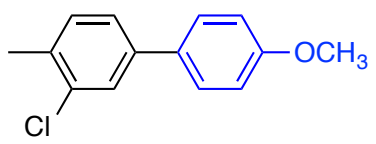

Colorless oil $(0.223 \mathrm{~g}, 96 \%)$

${ }^{1} \mathrm{H}$ NMR $\left(400 \mathrm{MHz}, \mathrm{DMSO}-\mathrm{d}_{6}\right) \delta: 7.63-7.59(\mathrm{~m}, 3 \mathrm{H}), 7.48(\mathrm{~d}, \mathrm{~J}=8.0 \mathrm{~Hz}, 1 \mathrm{H}), 7.37(\mathrm{~d}, \mathrm{~J}=7.6 \mathrm{~Hz}, 1 \mathrm{H}), 6.99$ $(\mathrm{d}, \mathrm{J}=8.4 \mathrm{~Hz}, 2 \mathrm{H}), 3.77(\mathrm{~s}, 3 \mathrm{H}), 2.33(\mathrm{~s}, 3 \mathrm{H})$.

Anal. Calcd for $\mathrm{C}_{14} \mathrm{H}_{13} \mathrm{ClO}$ : C, 72.26; $\mathrm{H}, 5.63$. Found: 72.18; $\mathrm{H}, 5.70$.

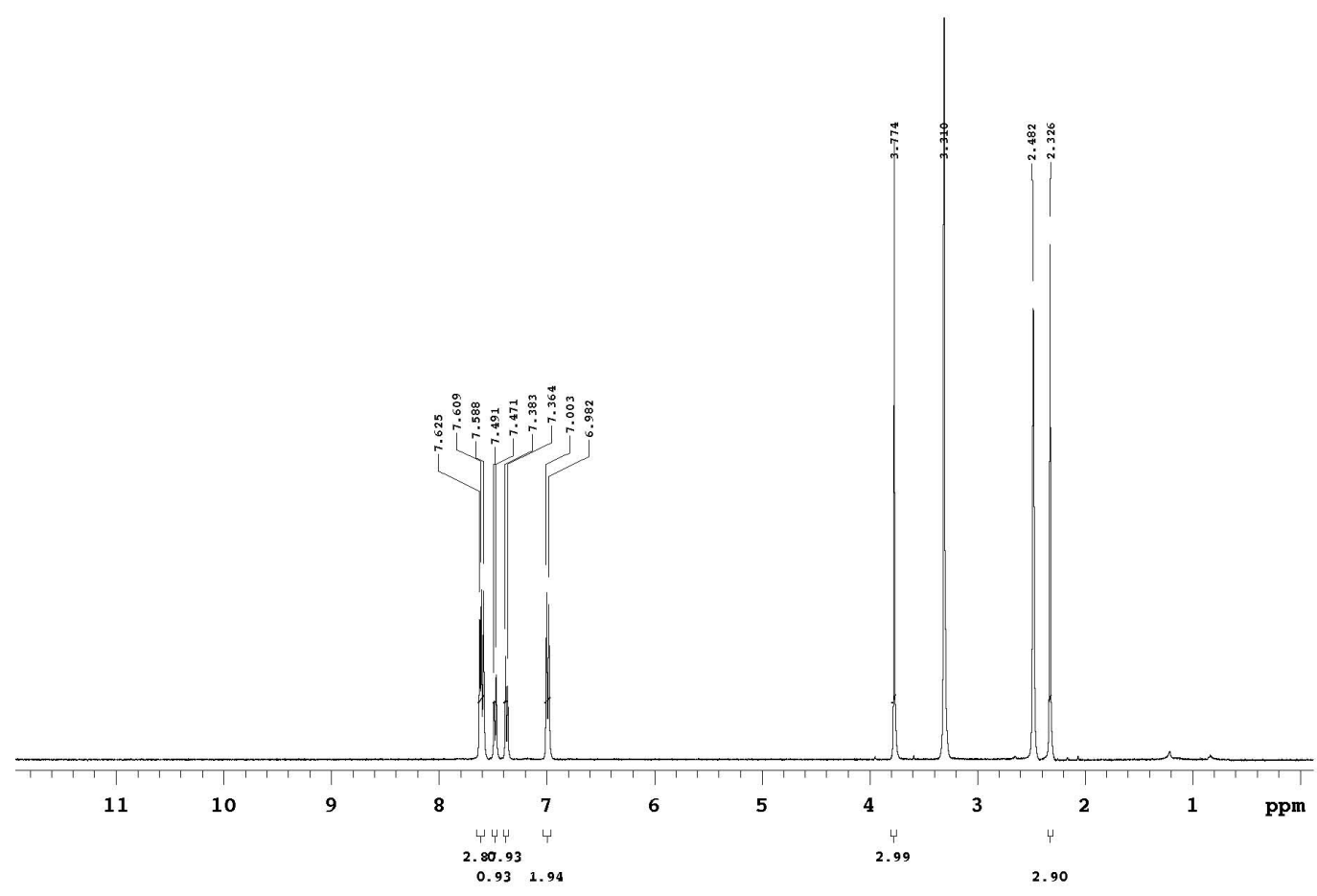


4-(4'-Methoxyphenyl)phenol (6m):<smiles>COc1ccc(-c2ccc(O)cc2)cc1</smiles>

White solid (0.194 g, 97\%); mp $181.5-182.2^{\circ} \mathrm{C}\left[\right.$ Lit. mp $\left.180-181^{\circ} \mathrm{C}\right] . .^{14}$

${ }^{1} \mathrm{H}$ NMR $\left(300 \mathrm{MHz}, \mathrm{DMSO}-\mathrm{d}_{6}\right) \delta: 9.41(\mathrm{~s}, 1 \mathrm{H}), 7.47(\mathrm{~d}, \mathrm{~J}=8.1 \mathrm{~Hz}, 2 \mathrm{H}), 7.39(\mathrm{~d}, \mathrm{~J}=8.7,2 \mathrm{H}), 6.95(\mathrm{~d}, \mathrm{~J}=8.7$ $\mathrm{Hz}, 2 \mathrm{H}), 6.79(\mathrm{~d}, \mathrm{~J}=9.0 \mathrm{~Hz}, 2 \mathrm{H}), 3.75(\mathrm{~s}, 3 \mathrm{H})$. Identical to ${ }^{1} \mathrm{H}$ NMR reported in the literature. ${ }^{15}$

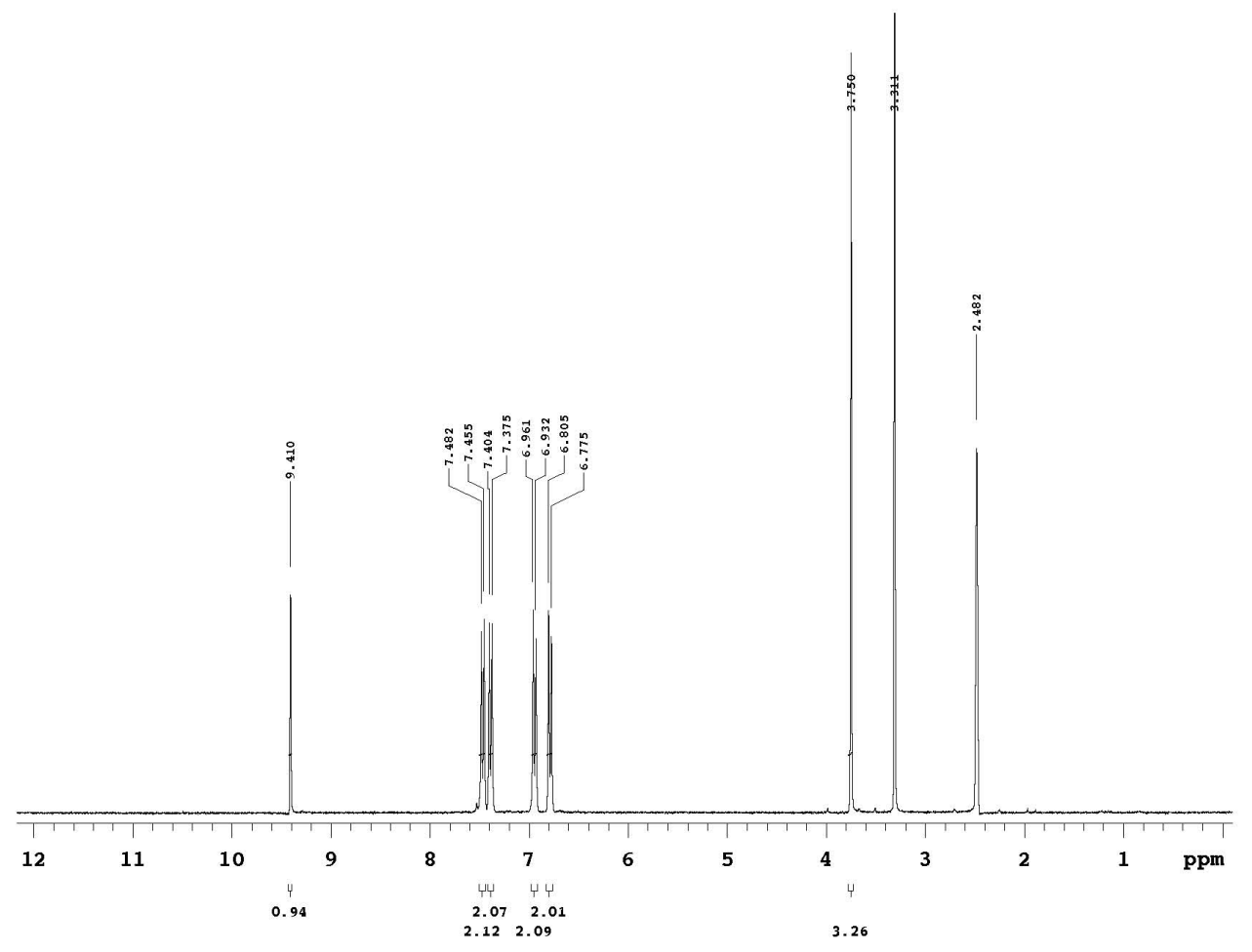




\section{(4-methoxyphenyl)aniline (6n)}

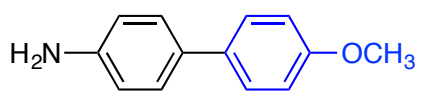

Light yellow solid (0.184 g, 92\%); mp 144.8-145.2 ${ }^{\circ} \mathrm{C}\left[\text { Lit. mp } 147^{\circ} \mathrm{C}\right]^{15}$

${ }^{1} \mathrm{H} \mathrm{NMR}\left(300 \mathrm{MHz}, \mathrm{DMSO}-d_{6}\right) \delta: 7.42(\mathrm{~d}, \mathrm{~J}=7.8 \mathrm{~Hz}, 2 \mathrm{H}), 7.26(\mathrm{~d}, \mathrm{~J}=7.2 \mathrm{~Hz}, 2 \mathrm{H}) 6.91(\mathrm{~d}, \mathrm{~J}=7.8 \mathrm{~Hz}, 2 \mathrm{H})$, $6.60(\mathrm{~d}, \mathrm{~J}=6.9 \mathrm{~Hz}, 2 \mathrm{H}), 5.10(\mathrm{~s}, 2 \mathrm{H}), 3.74(\mathrm{~s}, 3 \mathrm{H})$. Identical to ${ }^{1} \mathrm{H}$ NMR reported in the literature. ${ }^{16}$

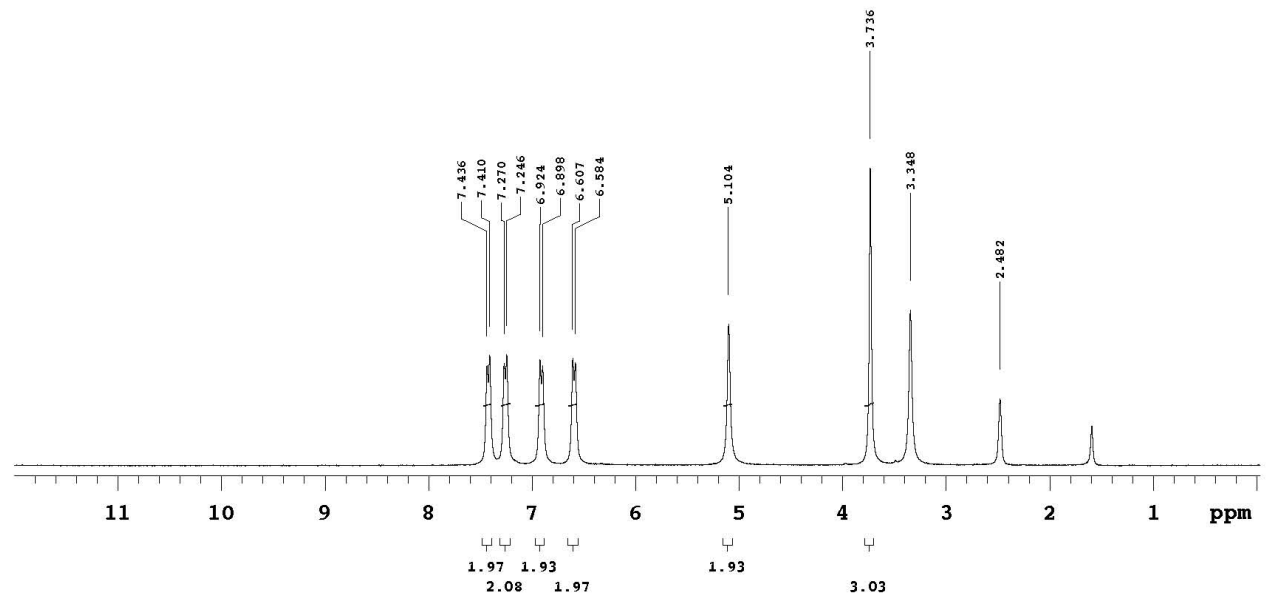




\section{References}

(1) Ackermann, L.; Althammer, A. Org. Lett. 2006, 8(16), 3457-3460.

(2) Lu, B.; Fu, C.; Ma, S. Tetrahedron Lett, 2010, 51(9), 1284-6

(3) Cepanec, I.; Litvic, M.; Udikovic, J.; Pogorelic, I.; Lovric, M. Tetrahedron 2007, 63 (25), 5614-5621.

(4) Alacid, E.; Najera, C. Org. Lett. 2008, 10(21), 5011-5014

(5) Kuriyama, M.; Shimazawa, R.; Shirai, R. Tetrahedron 2007, 63(38), 9393-9400.

(6) Roy, A. Hartwig, J. F. J. Am. Chem. Soc. 2003, 125, 8704-5.

(7). Doebele, M.; Vanderheiden, S.; Jung, N.; Brase, S. Angew. Chemie, Int. Ed. 2010, 49, 5986-5988.

(8) Alvarez-Bercedo, P.; Martin, R. J. Am. Chem. Soc. 2010, 132, 17352-17353.

(9) Guan, B.-T.; Wang, Y.; Li, B.J.; Yu, D.-G.; Shi, Z.-J. J. Am. Chem Soc. 2008, 130, 14468-14470.

(10) Wang, L.; Wang, Z.-X. . Org. Lett. 2007, 9(21), 4335-4338.

(11) Yu, D.-G.; Yu, M.; Guan, B.-T.; Li, B.-j.; Zheng, Y.; Wu, Z.-H.; Shi, Z.-J. Org. Lett. 2009, 11(15), 33743377.

(12) Molander, G. A.; lannazzo, L. J. Org. Chem. 2011, 76 (21), 9182-7.

(13) Gavryushin, A.; Kofink, C.; Manolikakes, G.; Knochel, P. Org. Lett. 2005, 7(22), 4871-4

(14) Abramovitch, R. A.; Alvernhe, G.; Bartnik, R.; Dassanayake, N. L.; Inbasekaran, M. N.; Kato, S. J. Am. Chem. Soc. 1981, 103(15), 4558-65.

(15) Razler, T. M.; Hsiao, Y.; Qian, F.; Fu, R.; Khan, R. K.; Doubleday, W. J. Org. Chem. 2009, 74(3), 1381-4.

(16) Scheuermann, G. M.; Rumi, L.; Steurer, P.; Bannwarth, W.; Mulhaupt, R. J. Am. Chem. Soc. 2009, 131(23), 8262-8270. 